Discussion Paper No. 965

\title{
GRADUAL ADJUSTMENT AND EQUILIBRIUM UNIQUENESS UNDER NOISY MONITORING
}

\author{
Ryota lijima \\ Akitada Kasahara \\ The $18^{\text {th }}$ ISER-Moriguchi Prize (2015) \\ Awarded Paper \\ March 2016 \\ The Institute of Social and Economic Research \\ Osaka University \\ 6-1 Mihogaoka, Ibaraki, Osaka 567-0047, Japan
}




\title{
Gradual Adjustment and Equilibrium Uniqueness under Noisy Monitoring*
}

\author{
Ryota Iijima and Akitada Kasahara ${ }^{\dagger}$
}

April 4, 2016

\begin{abstract}
We study the implications of flexible adjustment in strategic interactions using a class of finite-horizon models in continuous time. Players take costly actions to affect the evolution of state variables that are commonly observable and perturbed by Brownian noise. The values of these state variables influence players' terminal payoffs at the deadline, as well as their flow payoffs. In contrast to the static case, the equilibrium is unique under a general class of terminal payoff functions. Our characterization of the equilibrium builds on recent developments in the theory of backward stochastic differential equations (BSDEs). We use this tool to analyze applications, including team production, hold-up problems, and dynamic contests. In a team production model, the unique equilibrium selects an efficient outcome when frictions vanish.
\end{abstract}

[Keywords: continuous-time game, gradual adjustment, equilibrium uniqueness, coordination problem, team production, hold-up problem, contest]

${ }^{*}$ A previous version of this paper was circulated under the title "Gradualism and Equilibrium Uniqueness in Stochastic Differential Games." We are deeply grateful to Drew Fudenberg, Ben Golub, and Tomasz Strzalecki as well as Akihiko Takahashi for continuous encouragement and invaluable advice. We thank a co-editor and four referees for insightful and detailed comments that have substantially improved the paper. We also acknowledge helpful comments from Nageeb Ali, Darrell Duffie, Mira Frick, George Georgiadis, Johannes Hörner, Yuhta Ishii, Yuichiro Kamada, Kazuya Kamiya, Michihiro Kandori, David Laibson, Nicolas Lambert, Rida Laraki, Jonathan Libgober, Bart Lipman, Chiara Margaria, Eric Maskin, Akihiko Matsui, Stephen Morris, Nozomu Muto, Wojciech Olszewski, Daisuke Oyama, Larry Samuelson, Yuliy Sannikov, Andy Skrzypacz, Bruno Strulovici, Takuo Sugaya, Akihiko Takahashi, Masashi Toda, Zhe Wang, Daichi Yanagisawa, Jeff Zwiebel, and seminar/conference participants at various places. This paper was awarded the 18th Moriguchi Prize by the Institute of Social and Economic Research of Osaka University. All remaining errors are ours.

${ }^{\dagger}$ Iijima: Department of Economics, Harvard University, Email:riijima@fas.harvard.edu, and Kasahara: Graduate School of Business, Stanford University, Email:akitadak@stanford.edu. 


\section{Introduction}

In a variety of economic settings, strategic interactions are often modeled as one-shot games. According to this approach, time is not explicitly modeled and the outcome of a game is instantaneously determined as a consequence of players' static and simultaneous decisions. However, this approach abstracts away from the fact that players often take a certain amount of time to finalize their actions in real-world settings, such as investment or production. In these situations, players may want to gradually adjust their positions while additional noisy information arises. To examine the implications of such "gradual adjustment," we propose and analyze a broad class of finite-horizon games with stochastically evolving states.

As a motivating example, consider several firms engaged in a joint project with a fixed end date, such as the development of a new product with a pre-determined release date. Each firm continuously makes investments to improve the quality of the product, all the way up until the deadline. On the release date, demand for the new product is affected by market conditions and the overall quality of the product. If firms simultaneously choose their total investment levels at the start, then they face a coordination problem in the form of equilibrium multiplicity, especially when their decisions are strategic complements.

Our key insight in this study is that gradual adjustment eliminates this coordination problem: We show that a unique equilibrium can be obtained when firms can adjust their investment levels gradually. To formalize this insight, Section 2 builds a general class of continuous-time stochastic games which fit a variety of applied settings studied in the literature, including capital investment, sticky price competition, and $R \& D$ races. We consider a model with a fixed deadline and a commonly observed state variable whose evolution depends on players' actions and Brownian noise. Up until the deadline, players make flexible and frequent adjustments to the state by influencing the drift of the state variable process subject to convex adjustment costs. The costs can depend on the state variables (and can thus also be interpreted as a net flow payoff), and there is a lumpsum return that depends on the final values of the state variables at the deadline. This model can be interpreted as a dynamic elaboration of a one-shot game, in which players simultaneously and directly choose the values of the state variables. We solve for the Nash equilibrium of this class of games, allowing for history-dependent strategies.

In Section 3, we show that, under a general class of terminal payoff functions, the model has a unique equilibrium. Notably, this is true even if the adjustment costs and 
noise are arbitrarily small-and, perhaps more interestingly, if the corresponding oneshot model generates multiple equilibria. In addition, the unique equilibrium has the Markovian property, that is, each player's adjustment at each moment in time depends only on the current time and state and is independent of past histories.

We identify the following key assumptions needed for the uniqueness result: (i) gradual adjustment; (ii) bounded time horizon; (iii) no instantaneous strategic interdependence; and (iv) noisy state evolution. In the absence of any of these key assumptions, we can construct counterexamples where equilibrium multiplicity arises. For the basic intuition behind this, consider a discrete-period environment in which players commit to fixed actions during each period. In this environment, one's optimal choice of current action depends on the opponents' actions in the same period, which creates the potential for equilibrium multiplicity. However, this coordination incentive vanishes when the period length is very small. This follows from the fact that the opponents' current actions influence the state by only a small amount during any given period, and the continuation values are continuous in the state, because discontinuities are smoothed out by Brownian noise. Moreover, in any equilibrium the continuation values at the deadline are uniquely determined by the state variables, by definition. Given that payers' flow payoffs are independent of the opponents' current actions, these observations enable us to solve the game by "backward induction" and to uniquely compute the continuation values and optimal actions for earlier periods from those for the deadline in the limit.

Section 4 illustrates the scope of our general model by analyzing three specific applications. As a prime application, we consider a team production game in Section 4.1. Each player exerts costly effort to increase the value of a one-dimensional state variable, representing the team's total output, which generates a common-value benefit to the players at the deadline. There might exist multiple equilibria in the static benchmark case, even when the cost function is arbitrarily small. In our model, such a coordination failure disappears and there is a unique equilibrium. Moreover, provided the costs and noise are sufficiently small, this equilibrium is approximately efficient. Intuitively, this is because some noise realizations can substitute for current efforts on the part of the players and promote subsequent efforts on their part. When the state is sufficiently close to the socially efficient outcome near the deadline, the players can strongly coordinate on the efficient outcome with small costs and noise, which will unravel any inefficient equilibrium because the players will anticipate that even small noise fluctuations will trigger repeated 
coordination toward the efficient outcome.

In Section 4.2, we study a hold-up problem, in which a seller can improve the quality of a good but the price of the good is fixed ex-ante, independently of the quality. While the static benchmark case predicts no investment in quality, we show that in our setting with gradual adjustment the seller chooses positive investment levels. This illustrates a form of commitment power embedded in the gradually evolving state. We provide a closedform solution of the equilibrium strategies in this game. Finally, Section 4.3 studies a model of a dynamic contest, in which players exert costly effort to improve the quality of their own project, and a winner is chosen by comparing all players' quality levels at the deadline. In contrast to the static benchmark case, players have dynamic strategic incentives to discourage their opponents from making efforts in the future. This effect is non-stationary because of the deadline, and we quantify the size by numerically computing the equilibrium payoffs and strategies through partial differential equations (PDEs).

From a technical point of view, we employ techniques recently developed in the theory of backward stochastic differential equations (BSDEs). BSDEs are a form of (possibly non-Markovian) stochastic differential equation defined backwards in time from stochastic terminal conditions. The theory of BSDEs has been developed as a powerful approach to stochastic control problems in cases where the Hamilton-Jacobi-Bellman (HJB) equations are not applicable. To the best of our knowledge, this study is the first to use BSDEs to obtain equilibrium uniqueness in continuous-time games. ${ }^{1}$ In contrast to the standard approach in continuous-time games, which is based on recursive dynamic programming, we relate the optimality condition and equilibrium directly to BSDEs. As an important consequence, we do not need to require smoothness of the value functions, which is necessary the HJB equation approach but is often difficult to ensure, in particular in the case of multi-dimensional state variables. In addition, we apply the BSDE comparison theorem (Kobylanski, 2000) to obtain our efficiency result and other comparative statics in Section 4.1.

The Supplementary Appendix contains results for variations of the main model, as well as omitted proofs. In particular, we show, using a discrete-time model, that the uniqueness result holds under a Poisson noise environment as well if the period length is

\footnotetext{
${ }^{1}$ The theory of BSDEs was first introduced by Bismut (1973) for the linear case, and later by Pardoux and Peng (1990) for the uniform Lipschitz case. Duffie and Epstein (1992) used BSDE in constructing recursive utility in continuous time, and Chen and Epstein (2002) incorporate ambiguity aversion. Recently, Williams (2011) and Cvitanic, Wan, and Zhang (2009) used BSDEs in the context of dynamic contracts, while our focus is on strategic interactions among multiple long-run players.
} 
small enough.

\section{$1.1 \quad$ Related Literature}

We formulate a continuous-time model in which the players' actions are imperfectly monitored with Brownian signals as in Sannikov (2007). Our paper identifies situations in which the strategic interdependence during a short time interval is small, which implies the equilibrium uniqueness via the backward induction argument. This logic is different from several studies in the repeated-game literature that examine the role of Brownian signals in sustaining intertemporal incentives when actions are flexible. ${ }^{2}$ Sannikov and Skrzypacz (2010) show that Brownian signals are not effective for this purpose unless they are used for linear value transfers between agents. When such a transfer is not feasible because of structural restrictions, several studies have shown that equilibria are either static or Markovian when players' actions are flexible enough. For example, this is the case when there is only one long-run agent (Fudenberg and Levine, 2007, 2009; Faingold and Sannikov, 2011; Bohren, 2014) or a signal is one-dimensional (Sannikov and Skrzypacz, 2007). Those structural restrictions play no role in the uniqueness result in our paper. ${ }^{3}$

The model in our paper can be seen as a differential game in which flow payoffs are independent of the opponents' current actions. As we will see, the model is applicable to many economic examples in the literature (see Dockner, Jorgensen, Long, and Sorger (2001) for a survey). In important economic settings such as capital accumulation or duopoly with a sticky price, there typically exist many equilibria (Fudenberg and Tirole, 1983; Tsutsui and Mino, 1990). This paper contributes to the literature by providing a uniqueness result. Lepeltier, $\mathrm{Wu}$, and $\mathrm{Yu}$ (2009) and Hamadene and $\mathrm{Mu}$ (2015) consider imperfect monitoring models similar to ours, and use BSDEs to show equilibrium existence. In these models, flow payoffs do depend on the opponents' actions, which precludes equilibrium uniqueness in general. We also note that their proof method does not imply uniqueness, even under the setting in our paper. Papavassilopoulos and Cruz (1979) show that, in a certain class of deterministic models, an equilibrium is unique within the

\footnotetext{
${ }^{2}$ We choose an imperfect monitoring set-up in order to avoid technical issues that can arise in formalizing perfect monitoring in continuous time (e.g., Simon and Stinchcombe (1989) and Bergin and MacLeod (1993)). In Supplementary Appendix C.2.1, we show a sense in which the monitoring imperfectness itself is not important for our uniqueness result, by using a discrete-time model with noisy state evolution.

${ }^{3}$ We also note that the uniqueness result continues to hold in our model, even under Poisson noise (see Supplementary Appendix C.2.2), which is in sharp contrast to the findings of Fudenberg and Levine (2007) and Faingold and Sannikov (2011).
} 
class of Markov strategies that are analytic functions of the state, provided that such an equilibrium exists. ${ }^{4}$ We note that equilibrium strategies are often not differentiable even if there is noise. Also, discontinuity is an important source of equilibrium multiplicity, as we show in Example 3.

There are some studies on finite-horizon models with frequent actions that examine the effects of commitment power caused by switching or adjustment costs (most of these papers do not allow players to receive flow payoffs in addition to the costs). We note that a similar effect is at work in our model, because players cannot make appreciable changes in their state during a short time horizon, on account of the adjustment costs and the boundedness of the players' controls. Caruana and Einav (2008a) study Cournot competition using a deterministic differential game model with quadratic payoffs in which firms change the target production level before the market opens. In this study, we consider a much wider class of payoffs and costs under history-dependent strategies with imperfect public monitoring, focusing on equilibrium uniqueness. Lipman and Wang (2000) study finitely repeated games with finite actions and switching costs. They consider the effect of increasing the frequency of repetitions, which makes the switching cost disproportionally larger than a stage game payoff for each period. ${ }^{5}$ While they obtain an equilibrium selection result in binary-coordination games, the equilibrium is not unique in general.

In Kamada and Kandori (2014), players have opportunities to revise their positions simultaneously, at the times of arrival of synchronous Poisson shocks during the preparation phase. In contrast to our uniqueness result, they show that a broad range of outcomes can emerge as equilibria even if the static benchmark game has a unique equilibrium. In their model, adjustment is not gradual and can take any size, which makes it possible to implement effective punishments against deviations even when the remaining time is short. In contrast, our uniqueness result suggests that gradualness in adjustments can eliminate such punishment incentives during the preparation phase. Calcagno, Kamada, Lovo, and Sugaya (2014) consider a model of sequential moves that occur with asynchronous Poisson arrivals, and show that a unique equilibrium selects Pareto efficient outcome under a certain class of two-player coordination games. ${ }^{6}$

\footnotetext{
${ }^{4}$ Wernerfelt (1987) extends this result to a certain stochastic setting. That is, an equilibrium is unique within the class of Markov strategies that are $C^{2}$ in the state variables if such an equilibrium exists.

${ }^{5}$ To this effect, Caruana and Einav (2008b) analyze a model in which players announce their intended actions to be taken at the deadline and the switching cost of changing an action plan is increasing with time. Their model is a finite-period sequential-move game, and thus generically has a unique equilibrium.

${ }^{6}$ They provide an example of multiple equilibria when there are more than two players. Lovo and Tomala (2015) prove the existence of Makov perfect equilibria in a more general framework.
} 
Our focus is different from that of many papers that study gradual contributions to public goods. ${ }^{7}$ While the details of the models vary, the existing research typically questions whether and how gradual contributions can help to mitigate free-rider problems and focuses on the most efficient equilibrium among possible multiple equilibria. In contrast, we show how gradual adjustments and noise help achieve equilibrium uniqueness.

\section{Model}

\section{$2.1 \quad$ Setup}

We consider a continuous-time game over a finite horizon $[0, T]$ played by a finite set of players $N=\{1,2, \ldots, n\}$. Let $X_{t} \in \mathbb{R}^{d}$ denote the value of the $d$-dimensional state variable at time $t$. As we discuss in Section 2.2 below, the interpretation of this variable can vary with the application, representing for instance accumulated capital, project quality, or sticky price levels. The players gradually adjust the state variable subject to random shocks. Specifically, we assume $X_{t}$ follows a process of the form

$$
d X_{t}=\mu\left(A_{t}, X_{t}\right) d t+\Sigma d Z_{t}
$$

where $A_{t}=\left(A_{t}^{i}\right)_{i \in N}$ is the profile of control actions chosen by the players at time $t$, and $A_{t}^{i} \in \mathcal{A}^{i}$ is the control chosen by player $i$ at time $t .^{8}$ The control range $\mathcal{A}^{i}$ is assumed to be a compact interval in $\mathbb{R}$. Moreover, $Z_{t}=\left(Z_{t}^{k}\right)_{k=1, . ., d}$ is a $d$-dimensional Brownian motion, and $\Sigma$ is a $d \times d$ matrix that describes the correlation of the shocks to the state variable. The game starts with an exogenously given initial state $X_{0} \in \mathbb{R}^{d}$.

We impose the following restrictions on the state process in (1).

\section{Assumption 1.}

1. There exist $\mu_{i} \in \mathbb{R}^{d}$ for each $i \in N$ and a bounded, Lipschitz continuous function $\mu_{0}: \mathbb{R}^{d} \rightarrow \mathbb{R}^{d}$ such that $\mu(A, X)=\sum_{i \in N} \mu_{i} A^{i}+\mu_{0}(X)$.

2. The correlation matrix $\Sigma$ is invertible.

\footnotetext{
${ }^{7}$ For example, see Admati and Perry (1991), Fershtman and Nitzan (1991), Marx and Matthews (2000), Lockwood and Thomas (2002), Compte and Jehiel (2004), Pitchford and Snyder (2004), Matthews (2013), and Battaglini, Nunnari, and Palfrey (2014). Guéron (2015) considers a discrete-time infinite horizon model and obtains an equilibrium uniqueness result under an imperfect monitoring set-up.

${ }^{8}$ We use the so-called weak formulation, which is the standard way that a continuous-time game with imperfect monitoring is formulated in the literature (see Appendix B.2 for more formal description).
} 
The first condition requires that the drift term be additively separable across players and linear in their actions. As we will see, the separability ensures that each player's instantaneous control choice at any given moment is independent of the control choices of the other players at that moment, which is important for equilibrium uniqueness. The second condition rules out perfect correlation among the components of the state variables.

At each time $t$, player $i$ incurs an instantaneous adjustment cost $c^{i}\left(A_{t}^{i}, X_{t}\right) d t$, where $c^{i}: \mathcal{A}^{i} \times \mathbb{R}^{d} \rightarrow \mathbb{R}$. At the end of the game, that is, at time $T$, each player receives a terminal payoff $U^{i}: \mathbb{R}^{d} \rightarrow \mathbb{R}$ as a function of the terminal state $X_{T}$. To summarize, player $i$ 's total payoff is

$$
U^{i}\left(X_{T}\right)-\int_{0}^{T} c^{i}\left(A_{t}^{i}, X_{t}\right) d t
$$

We impose the following conditions on $c^{i}$ and $U^{i}$.

\section{Assumption 2.}

1. $c^{i}$ is $C^{2}$ and has bounded derivatives such that $\inf _{A^{i}, X} \frac{\partial^{2} c^{i}\left(A^{i}, X\right)}{\left(\partial A^{i}\right)^{2}}>0$.

2. $U^{i}$ is bounded and Lipschitz continuous.

The first condition ensures that the adjustment cost is strictly convex in the control size. The second condition is a technical requirement.

At time $t$, player $i$ observes the state $X_{t}$ but does not directly observe the control actions $A_{t}^{j}$ of the other players. Formally, player $i$ 's (public) strategy is an $\mathcal{A}^{i}$-valued stochastic process $A^{i}=\left(A_{t}^{i}\right)_{t \in[0, T]}$ that is progressively measurable with respect to $\mathcal{F}^{X}$, the filtration generated by the public history of $X_{t}$. Note that this setup allows the players' actions to depend on past histories of the $X_{t}$ process.

Under any profile of feasible strategies $A=\left(A^{i}\right)_{i \in N}$, we can define player $i$ 's continuation value at time $t$ as

$$
W_{t}^{i}(A)=\mathbb{E}\left[U^{i}\left(X_{T}\right)-\int_{t}^{T} c^{i}\left(A_{s}^{i}, X_{s}\right) d s \mid \mathcal{F}_{t}^{X}\right]
$$

A profile of feasible strategies $A=\left(A^{i}\right)_{i \in N}$ is a Nash equilibrium if each player maximizes her expected payoff (2) at $t=0$ given the other players' strategies. Since the distribution of the publicly observable $X_{t}$ process has full support in our setup, the Nash equilibrium concept is identical to its refinement known as public perfect equilibrium, which is frequently used in the literature on dynamic games. 


\subsection{Discussion of the Model}

In this subsection, we discuss two interpretations of the model. Our main motivation is to study the effects of introducing a flexible adjustment phase into a static game framework. To see this, consider the special case in which $c^{i}$ does not depend on $X_{t}$ and $\mu_{0}$ is identically 0. Such a game can be viewed as a "dynamic elaboration" of the following static game: 9

Player $i$ chooses $A_{0}^{i} \in \mathcal{A}^{i}$ at time 0 and receives a payoff of $U^{i}\left(X_{T}\right)-T c^{i}\left(A_{0}^{i}\right)$

at time $T$, where $X_{T}=X_{0}+T \sum_{i} \mu_{i} A_{0}^{i} \cdot{ }^{10}$

In this game, player $i$ can be seen as committing to the particular control level $A_{0}^{i}$ during the entire interval $[0, T]$. Without substantial restrictions on the functional forms of the $U^{i}$, this static game can have multiple Nash equilibria. Our model elaborates on this static game by introducing a noise term $Z_{t}$ and allowing players to change their action $A_{t}^{i}$ during the preparation phase $[0, T]$. As shown in the next section, this natural modification leads to equilibrium uniqueness under a broad class of terminal payoff functions. Moreover, because the state is observable during the preparation phase $[0, T]$, players are faced with dynamic strategic interactions that are absent in the static benchmark. We analyze this dynamic aspect in Section 4, within the context of three applications, namely team production, hold-up problems, and dynamic contests.

Alternatively, we can interpret $-c^{i}$ as player $i$ 's net flow payoff. Under this interpretation, our model accommodates many games studied in the literature on differential games, including the two examples given below. (See Dockner, Jorgensen, Long, and Sorger (2001) for other examples.) We note that the papers mentioned below consider a stationary environment without noise, and thus do not obtain equilibrium uniqueness.

Example 1 (Capital accumulation). Consider firms competing to expand their capital levels. Let $d=n$, so that the number of players coincides with the dimension of the state variable. $X_{t}^{i}$ is the capital level of firm $i$, which follows $d X_{t}^{i}=A^{i} d t+d Z_{t}^{i}$, and $A_{t}^{i}$ is the net investment made by $i$ at time $t$. Each firm receives flow revenue as a function of the profile of the current capital levels $X_{t}$, and incurs a flow investment cost that is convex in $A_{t}^{i}$. Thus $c^{i}\left(A_{t}^{i}, X_{t}\right)$ represents the flow revenue minus the investment cost at time $t$. Spence (1979) proposes this class of games, and Fudenberg and Tirole (1983) show

\footnotetext{
${ }^{9}$ Similarly, Caruana and Einav (2008a,b), Kamada and Kandori (2014) and Calcagno, Kamada, Lovo, and Sugaya (2014) analyze games in which players adjust their plans for future actions. The relationship between these studies and ours is more extensively discussed in the Related Literature section.

${ }^{10} \mathrm{An}$ alternative but similar formulation of a corresponding static game includes a noise term by setting $X_{T}=X_{0}+T \sum_{i} \mu_{i} A_{0}^{i}+\Sigma Z_{T}$. Similarly, multiple equilibria are possible with this formulation.
} 
that there exists a continuum of multiple (Markov perfect) equilibria, in contrast to our uniqueness result with a fixed horizon and noise.

Example 2 (Duopoly with a sticky price). Fershtman and Kamien (1987) propose a model of duopolistic competition in a homogeneous good whose price adjusts gradually. ${ }^{11}$ Our framework extends their model by introducing noise (due to noise traders, for example) and using a one-dimensional state $X_{t}$ as the price of the good at time $t$. $A_{t}^{i}$ represents firm $i$ 's supply at time $t$. The price adjustment takes the form $d X_{t}=$ $\left(\bar{X}-\sum_{i} A_{t}^{i}-X_{t}\right) d t+d Z_{t}$, where $\bar{X}$ is a fixed parameter. Here $\bar{X}-\sum_{i} A_{t}^{i}$ denotes the price level induced by a linear demand curve based on the current output $\sum_{i} A_{t}^{i}$. The flow payoff for firm $i$ at time $t$, which consists of the revenue minus the production cost, is independent of $\left(A_{t}^{j}\right)_{j \neq i}$ since the current price $X_{t}$ is unaffected by the firms' current output choices.

\section{Equilibrium Uniqueness}

In this section, we establish equilibrium uniqueness. Section 3.1 states the formal result, while Section 3.2 provides intuition for the result, and Section 3.3 discusses the role of our key assumptions.

\subsection{Uniqueness Result}

We follow DeMarzo and Sannikov (2006) in characterizing the optimality of each player's strategy by using the martingale representation theorem (e.g., Karatzas and Shreve (1991)). For any profile of feasible policies $A=\left(A^{i}\right)_{i \in N}$, the evolution of player $i$ 's continuation value satisfies

$$
d W_{t}^{i}(A)=c^{i}\left(A_{t}^{i}, X_{t}\right) d t+\beta_{t}^{i}(A) \cdot d Z_{t}
$$

along with the terminal condition

$$
W_{T}^{i}(A)=U^{i}\left(X_{T}\right)
$$

\footnotetext{
${ }^{11}$ Tsutsui and Mino (1990) construct multiple equilibria of this game.
} 
Here $\beta^{i}(A)$, whose existence and uniqueness are ensured by the martingale representation, is an $\mathcal{F}^{X}$-progressively measurable $d$-dimensional process such that $\mathbb{E}\left[\int_{0}^{T}\left|\beta_{t}^{i}\right|^{2} d t\right]<\infty$. $^{12}$ Intuitively, $\beta_{t}^{i, k}(A)$ measures the sensitivity of player $i$ 's continuation payoff to fluctuations in $Z_{t}^{k}$ at time $t$.

Rewriting $d X_{t}=\mu\left(A_{t}, X_{t}\right) d t+\Sigma d Z_{t}$ as $d Z_{t}=\Sigma^{-1}\left(d X_{t}-\mu\left(A_{t}, X_{t}\right) d t\right)$ and substituting the latter into equation (3), we obtain

$$
d W_{t}^{i}(A)=\left[c^{i}\left(A_{t}^{i}, X_{t}\right)-\beta_{t}^{i}(A) \cdot \Sigma^{-1}\left(\sum_{j} \mu_{j} A_{t}^{j}+\mu_{0}\left(X_{t}\right)\right)\right] d t+\beta_{t}^{i}(A) \cdot \Sigma^{-1} d X_{t}
$$

where we used Assumption 1. To characterize the players' incentive compatibility constraint, we define player $i$ 's response function $f^{i}: \mathbb{R}^{n \times d} \times \mathbb{R}^{d} \rightarrow \mathcal{A}^{i}$ as

$$
f^{i}(b, x)=\underset{\alpha^{i} \in \mathcal{A}^{i}}{\arg \max }\left\{\left(b^{i} \cdot \Sigma^{-1} \mu_{i} \alpha^{i}\right)-c^{i}\left(\alpha^{i}, x\right)\right\}
$$

for each $b=\left(b^{i}\right)_{i \in N} \in \mathbb{R}^{n \times d}$ and $x \in \mathbb{R}^{d}$. The maximand is taken from (the negative of) the drift term in the expression for the continuation value above. The function $f^{i}$ is well-defined because we assume strict convexity of $c^{i}$ in $A^{i}$ (Assumption 2). Using these response functions, the optimality of the strategies can be characterized in terms of the following "local incentive compatibility condition" (Lemma 7 in Appendix): $i$ 's strategy $A^{i}$ is optimal against $A^{-i}$ if and only if

$$
A_{t}^{i}=f^{i}\left(\beta_{t}(A), X_{t}\right) \text { almost surely for almost all } t
$$

Lemma 1. A strategy profile $\left(A^{i}\right)_{i \in N}$ is a Nash equilibrium if and only if (3), (4), and (5) hold for some $\mathcal{F}^{X}$-progressively measurable processes $W, \beta$ such that

$$
\mathbb{E}\left[\sup _{0 \leq t \leq T}\left|W_{t}\right|^{2}\right], \mathbb{E}\left[\int_{0}^{T}\left|\beta_{t}\right|^{2} d t\right]<\infty
$$

Combining equations (1), (3), (4), and (5) leads to a multi-dimensional backward stochastic differential equation (BSDE)

$$
\begin{aligned}
& d W_{t}^{i}=\left[c^{i}\left(f^{i}\left(\beta_{t}, X_{t}\right)\right)-\beta_{t}^{i} \cdot \Sigma^{-1}\left(\sum_{j} \mu_{j}\left(X_{t}\right) f^{j}\left(\beta_{t}, X_{t}\right)+\mu_{0}\left(X_{t}\right)\right)\right] d t+\beta_{t}^{i} \cdot \Sigma^{-1} d X_{t} \\
& W_{T}^{i}=U^{i}\left(X_{T}\right)
\end{aligned}
$$

\footnotetext{
${ }^{12}$ In this paper we use $|\cdot|$ to denote both the Euclidean norm for vectors and the Frobenius norm for matrices.
} 
under a probability measure for which the $X_{t}$ process is a Brownian motion. ${ }^{13} \mathrm{~A}$ solution $(W, \beta)$ to this BSDE describes the stochastic evolution of the continuation payoffs. Moreover, the control $A_{t}^{i}$ given by $f^{i}\left(\beta_{t}, X_{t}\right)$ is incentive compatible for player $i$ and is consistent with the evolution of the continuation payoffs.

We build on the results of Delarue (2002) and Cheridito and Nam (Forthcoming) to show that this BSDE has a unique solution $(W, \beta)$, which establishes our main claim of equilibrium uniqueness.

Theorem 1. Under Assumptions 1 and 2, there exists a unique Nash equilibrium. Moreover, this equilibrium has the Markovian property, that is, the continuation values and the strategies take the form $W_{t}^{i}(A)=w^{i}\left(t, X_{t}\right)$ and $A_{t}^{i}=a^{i}\left(t, X_{t}\right)$ for some function $w^{i}:[0, T] \times \mathbb{R}^{d} \rightarrow \mathbb{R}$ that is Lipschitz continuous in $x \in \mathbb{R}^{d}$ uniformly in $t \in[0, T]$ and some function $a^{i}:[0, T] \times \mathbb{R}^{d} \rightarrow \mathcal{A}^{i}$.

The intuition underlying the uniqueness result and the key assumptions on which it relies are discussed in detail in Sections 3.2 and 3.3. Recall that we allow players' actions to depend on past histories of $X_{t}$ in a non-trivial manner. However, by Theorem 1 the unique equilibrium turns out to be Markovian.

\subsection{Heuristic Explanation of Uniqueness}

To glean some intuition for the uniqueness result (Theorem 1), consider a discrete-time version of the model with a short period length $\Delta$. This departs from the original model only in that each player's action remains constant within each discrete period. To simplify the notation, we assume that $d=1$ and $\Sigma=1$. At the start of the final period, $t=T-\Delta$, player $i$ chooses $A_{t}^{i}$ to maximize the value of

$$
\begin{aligned}
W_{t}^{i, \Delta} & =-c^{i}\left(A_{t}^{i}, X_{t}\right) \Delta+\mathbb{E}\left[U^{i}\left(X_{T}\right) \mid X_{t}\right] \\
& =-c^{i}\left(A_{t}^{i}, X_{t}\right) \Delta+\mathbb{E}\left[U^{i}\left(X_{t}+\left(\sum_{j \in N} \mu_{j} A_{t}^{j}+\mu_{0}\left(X_{t}\right)\right) \Delta+\epsilon_{t}\right) \mid X_{t}\right]
\end{aligned}
$$

given the opponents' actions $\left(A_{t}^{j}\right)_{j \neq i}$, where $\epsilon_{t}$ is distributed $N(0, \Delta)$. Note that the second term depends on the actions of all the players, $\left(A_{t}^{i}\right)_{i \in N}$. It is precisely because of this strategic interdependence that the subgame at the last period can have multiple

\footnotetext{
${ }^{13}$ See Appendix B.1.1 for a formal description.
} 
equilibria when $\Delta$ is not small.

We claim that if $\Delta$ is sufficiently small, then the strategic interdependence is "small" and the equilibrium action profile at $t=T-\Delta$ is unique. To see this, for now assume that $U^{i}$ is twice differentiable. Then we can directly compute

$$
\frac{\partial^{2}}{\partial A_{t}^{i}, \partial A_{t}^{j}} \mathbb{E}\left[U^{i}\left(X_{T}\right) \mid X_{t}\right]=\mathbb{E}\left[\left(U^{i}\right)^{\prime \prime}\left(X_{T}\right) \mid X_{t}\right] \mu_{i} \mu_{j} \Delta^{2}=O\left(\Delta^{2}\right)
$$

Intuitively speaking, this is because each player's action influences the state to first order in $\Delta$, and the interactions among their influences is of order less than $\Delta$, since the drift in the value of the state variable is separable across players. On the other hand, the secondorder partial derivative $\frac{\partial^{2}}{\partial\left(A_{t}^{i}\right)^{2}} W_{t}^{i, \Delta}$ is of the order of $\Delta$ because of the strict convexity of the adjustment cost. The first-order condition implicitly defines $i$ 's best response against the opponents' actions at time $t$. By the implicit function theorem, its slope is bounded by

$$
\left|\frac{\frac{\partial^{2}}{\partial A_{t}^{i} \partial A_{t}^{j}} W_{t}^{i, \Delta}}{\frac{\partial^{2}}{\partial\left(A_{t}^{i}\right)^{2}} W_{t}^{i, \Delta}}\right|,
$$

which is close to 0 when $\Delta$ is small. This means that each player's incentive is nearly insensitive to her opponents' actions, and thus the equilibrium actions at time $t$ are uniquely determined by the contraction mapping theorem.

In the above argument, the presence of noise becomes important when $U^{i}$ is not sufficiently smooth, because it is this noise that ensures the differentiability of $\mathbb{E}\left[U^{i}\left(X_{T}\right) \mid X_{t}\right]$ in $\left(A^{j}\right)_{j \in N}$ by convolution. ${ }^{14}$ The convexity of $c^{i}$ ensures that each player's incentive problem is a well-behaved concave problem for small $\Delta$, which implies that the best-response function is continuous. Moreover, the continuation value at $t$ depends on only the current state $X_{t}$, and is Lipschitz continuous by the implicit function theorem. We can then repeat this argument backward from the final period to recursively pin down the continuation values. $^{15}$

\subsection{Key Assumptions Underlying the Uniqueness}

In this subsection, we identity four key assumptions underlying the result:

\footnotetext{
${ }^{14}$ In general, this term is smooth even when $U^{i}$ is discontinuous. When $U^{i}$ is only Lipschitz continuous, we can show that $\frac{\partial^{2}}{\partial A_{t}^{i}, \partial A_{t}^{j}} \mathbb{E}\left[U^{i}\left(X_{T}\right) \mid X_{t}\right]=O\left(\Delta^{3 / 2}\right)$. See Appendix B.4 for the details.

${ }^{15}$ Strictly speaking, the Lipschitz coefficient should not grow too fast as we repeat the backward induction steps to continue this argument. See Supplementary Appendix C.2.1 for the details.
} 
(i) Gradual adjustment

(ii) Bounded time horizon

(iii) No instantaneous strategic dependence

(iv) Noisy state evolution

By the term "gradual adjustment" in (i), we mean that a player's decision at each time has only a small influence on the value of the state variable. In the discrete-time model discussed in Section 3.2, this influence is of order $\Delta$. In the case of the Poisson noise model (see Supplementary Appendix C.2.2), it is also of order $\Delta$ in expectation, and we prove that there exists a unique equilibrium if $\Delta$ is small enough. If $\Delta$ is large, we cannot ensure uniqueness in general, for the same reason that a one-shot game can have multiple equilibria. To understand why (ii) is necessary, consider the case in which the terminal deadline $T$ is randomly distributed and has unbounded support. Then it is not possible to apply the "backward induction" argument, which requires a definite terminal date. (See Supplementary Appendix C.1 for an explicit construction of multiple equilibria in the case of a random time horizon with an unbounded distribution.) By (iii), we mean the additive separability of the drift term of the $X_{t}$-process and the fact that $c^{i}$ does not depend on the current actions of the other players, $\left(A_{t}^{j}\right)_{j \neq i}$. When (iii) is violated, each player's local incentive constraint $\arg \max _{\alpha^{i} \in \mathcal{A}^{i}}\left\{\left(\beta^{i} \cdot \Sigma^{-1} \mu\left(\alpha^{i}, A^{-i}, X_{t}\right)\right)-c^{i}\left(\alpha^{i}, A^{-i}, X_{t}\right)\right\}$ defines an "augmented game" that, given $(\beta, X)$, does not in general uniquely pin down the optimal action profile. ${ }^{16}$ For (iv), recall that as established by Theorem 1, the continuation value functions become continuous in the state variable in the presence of Brownian noise. Without noise, it is possible that continuation value functions are discontinuous in states. This can lead to a continuum of multiple equilibria, as in Example 3 (Section 4.1). We also note that there is a technical issue in formulating non-Markovian strategies without noise in continuous time (e.g., Simon and Stinchcombe (1989)).

\footnotetext{
${ }^{16}$ Even when flow payoffs directly depend on the actions of others, we can generalize the equilibrium uniqueness result if every pair $(\beta, X)$ induces a unique enforceable action profile in the sense of Sannikov (2007), and this unique profile is Lipschitz continuous in $\beta$ and $X$. For example, this is the case when the payoff is an additively separable function of opponents' actions and own action. The same argument applies to the case in which the drift of the $X_{t}$ process is not separable across players. Analogous conditions appear as Condition 2 in Faingold and Sannikov (2011) and Assumption 4 in Bohren (2014), and these are used to rule out multiple Markov equilibria in games between a long-run agent and a sequence of short-run agents.
} 


\section{Applications}

In this section we study three applications of the model. Unless stated explicitly, we suppose Assumptions 1 and 2.

\subsection{Team Production}

We consider a team production model in which the state variable $X_{t}$ is one-dimensional as follows:

$$
d X_{t}=\sum_{i \in N} A_{t}^{i} d t+\sigma d Z_{t}
$$

where $\sigma>0$ and $Z_{t}$ is a one-dimensional Brownian motion. The state variable can be interpreted as, for example, the total production level by a team of workers, the amount of a public good, or the quality level of a joint research project. Players receive a common terminal payoff, denoted by $U: \mathbb{R} \rightarrow \mathbb{R}$. They also have the same cost function $\kappa c(\cdot) \geq 0$, with $c: \mathcal{A} \rightarrow \mathbb{R}$ and a scaling parameter $\kappa>0$, where $\mathcal{A}=[\underline{A}, \bar{A}]$ is the control range that is common across the players. ${ }^{17}$

Before analyzing this model, we will demonstrate the issue of multiple equilibria and inefficiency under benchmark cases. The following simple example shows equilibrium multiplicity under both the static benchmark and the deterministic case $\sigma=0$ even if $\kappa$ is arbitrarily small.

Example 3. Consider a form of "debt contract" $U(X)=\max \{0, X\}$. That is, players receive a positive benefit if their total output is positive and zero payoff otherwise. Assume that $\mathcal{A}=[0,1]$ and $c$ is strictly increasing. Also, suppose that $T<-X_{0}<N T$.

1. Consider the static benchmark model, in which player $i$ chooses effort level $A_{0}^{i}$ only once and the final output is determined by $X_{T}=X_{0}+T \sum_{i} A_{0}^{i}$ (Section 2.2). If $\kappa c^{\prime}(1)<1$, then there exists a Nash equilibrium $A_{0}^{i}=1$ for every $i$. The symmetric equilibrium payoff is $X_{0}+T N-T \kappa c(1)$. On the other hand, there is always a Nash equilibrium in which $A_{0}^{i}=0$ for every $i$. In this case, the symmetric equilibrium payoff is $X_{0}-T \kappa c(0)$. Note that the equilibrium outcome in the latter case is Pareto dominated by that in the former case. ${ }^{18}$

\footnotetext{
${ }^{17}$ See Radner (1992) and Zandt (1999) for surveys of models of team production. Georgiadis (2015) and Cvitanic and Georgiadis (2015) study Markov equilibria in a continuous-time model similar to ours in which the game terminates if and only if the state variable reaches some pre-established threshold.

${ }^{18}$ Even if we consider the alternative formulation of the static benchmark model with noise, that is, $X_{T}=$ $X_{0}+T \sum_{i} A_{0}^{i}+\sigma Z_{T}$, these two strategy profiles remain Nash equilibria when $\sigma$ is small.
} 
2. The equilibrium multiplicity in the static benchmark model carries over to the case where time is continuous but there is no noise. Consider a variation of the main model in which the noise level $\sigma$ is 0 , and focus on Markov strategies in which the control at time $t$ depends on only $t$ and $X_{t}$. If $N \geq 3$, then for any $\theta \in(-N+1,-1)$, the symmetric strategy profile in which player $i$ chooses control

$$
A_{t}^{i}= \begin{cases}1 & \text { if } X_{t} \geq(T-t) \theta \\ 0 & \text { if } X_{t}<(T-t) \theta\end{cases}
$$

is a Markov perfect equilibrium (for the details, see Appendix B.5). Thus, for a fixed initial state, there is a continuum of equilibria parameterized by $\theta$. If $X_{0} \geq T \theta$, the players always choose the maximal effort $A_{t}^{i}=1$ on the equilibrium path, and they achieve the symmetric equilibrium payoff $X_{0}+T N-T \kappa c(1)$. If $X_{0}<T \theta$, there is no effort at all, that is, $A_{t}^{i}=0$, and the symmetric equilibrium payoff is $X_{0}-T \kappa c(0)$, which is lower than the former case. Note that, in this class of equilibria, the continuation value of each player is discontinuous at this state. ${ }^{19}$

The main model predicts a unique Nash equilibrium under arbitrary values of $\kappa>0$ and $\sigma>0$. Below we provide some results that partially characterize the unique equilibrium payoff that is common across players. Our approach to the proofs of these results, which is based on the BSDE comparison theorem (Kobylanski, 2000) that compares the solutions of two different BSDEs, is of independent interest.

First, we provide (rough) bounds of the unique equilibrium payoff, and use these to characterize the limit equilibrium when noise and costs are small. The idea is to construct a hypothetical single-agent problem in which the agent chooses all the players' strategies. Formally, consider the problem of choosing a strategy profile $\hat{A}=\left(\hat{A}^{i}\right)_{i \in N}$ to maximize $\mathbb{E}\left[U\left(X_{T}\right)-\int_{0}^{T} \kappa \sum_{i} \hat{c}\left(\hat{A}_{t}^{i}\right) d t\right]$. Let $\hat{W}_{0}$ denote the optimal value. Let $W_{0}$ denote the equilibrium payoff under the unique equilibrium, which is symmetric across players. The following result provides two-sided bounds on the equilibrium payoff:

Lemma 2. Consider the team production game. Then $\hat{W}_{0}+\kappa T(N-1) \bar{c} \geq W_{0} \geq \hat{W}_{0}$.

\footnotetext{
${ }^{19}$ The nature of multiplicity is similar to that of Fudenberg and Tirole (1983) under an infinite-horizon capacity expansion game (Example 1), in that discontinuities in the continuation value functions play a key role.
} 
We compare the BSDEs under the equilibrium and the representative agent problem. The bounds become tight when $\kappa$ is small. We can show that, under small frictions, the representative agent approximately achieves the efficient payoff $\sup _{x \in\left[X_{0}+N T \underline{A}, X_{0}+N T \bar{A}\right]} U(x)$, which provides an accurate lower bound on the equilibrium payoff. As a consequence, the equilibrium is shown to achieve approximate efficiency under small frictions.

Proposition 1. Consider the team production game. Then, for any $\epsilon>0$, there exists $\delta>0$ such that

$$
\mathbb{E}\left[U\left(X_{T}\right)\right] \geq \max _{x \in\left[X_{0}+N T \underline{A}, X_{0}+N T \bar{A}\right]} U(x)-\epsilon
$$

holds under the unique equilibrium if $\sigma, \kappa \leq \delta$.

Recall that there can be an inefficient equilibrium even when $\kappa$ is small if there is no noise (Example 3). Proposition 1 ensures that such a coordination failure cannot occur in our dynamic setting when frictions are sufficiently small. To informally explain why inefficient equilibria (as in the above example) do not exist, consider the case in which the state is sufficiently close to the socially efficient outcome near the deadline. Then the players can coordinate on the efficient outcome and they attain high continuation payoffs at this state. This triggers players to coordinate to target toward the efficient state from nearby states in earlier times. The value of the state can eventually end up near an inefficient local maximum by an "unlucky" noise realization. In such a case, players might want to coordinate on the inefficient local maximum instead if the time left until the deadline is insufficient to push the state toward the most efficient one. However, the probability that such an event will occur shrinks to 0 if the noise level goes to 0 .

To examine the dependence of the equilibrium outcome distribution on the initial point $X_{0}$ when frictions are not necessarily small, we conducted numerical simulations. We specified $U(x)=\sin (a x)+b x-c x^{2}$ with $a=0.5$, and $b=c=0.01$ (Figure 1), and used the quadratic cost function $c(a)=\frac{\kappa}{2} a^{2}$. There are two local maxima, and we cannot rule out multiple equilibria in the static benchmark case, because the players can coordinate on "targeting" either of the locally maximum points. In particular, the players might coordinate on playing a Pareto-inefficient equilibrium by targeting the local maximum at $x \approx-8.30$ rather than the global maximum at $x \approx 3.28$.

Figure 2 shows a set of simulation results for the stated utility function with different initial points $X_{0}$ and cost levels $\kappa$. In each panel, we fix $X_{0} \in\{-6,0,-5.2\}$ and compare the simulated distribution of $X_{T}$ with $\kappa=0.5$ (grey) and that with 0.2 (black). For each parameter specification, we compute the equilibrium strategy profile and then randomly 
generated $X_{T} 1000$ times and plotted the resulting values as a histogram. We found that when $X_{0}$ is very close to one of the local maxima of $U\left(X_{T}\right)$, as in Panels A and $\mathrm{B}$, the players tend to coordinate on the closer of the two local maxima, regardless of cost level. However, when the initial point is not very close to one of the local maxima, the cost level can significantly affect the distribution of $X_{T}$. In Panel C, although $X_{0}=-5.2$ is closer to the inefficient local maximum, players try to push the state variable toward the global maximum in more than half of the simulations when the cost level is sufficiently small. ${ }^{20}$

Finally, we consider comparative statics of the equilibrium payoff assuming that $U$ is monotone. We let $A(\kappa, N)$ denote the unique equilibrium under the cost parameter $\kappa$ and team size $N$.

Proposition 2. Consider the team production game in which $U$ is increasing. Then $W_{0}(A(\kappa, N))$ is decreasing in $\kappa$ and increasing in $N$.

While this result may not be surprising in and of itself, it ensures that our "equilibrium selection" has natural properties. In general, an arbitrary selection of an equilibrium can lead to strange comparative statics results when we vary parameters locally. The proof is based on the comparison theorem applied to the equilibrium BSDEs across different parameters. In order to conduct the appropriate comparison, we need to ensure that $\beta_{t}$ is always positive in the equilibrium. This is guaranteed by the co-monotonicity theorem (Chen, Kulperger, and Wei, 2005; dos Reis and dos Reis, 2013), which is useful in checking the signs of sensitivity processes $\beta_{t}$ of BSDEs in general.

Remark 1. By the uniqueness result for a one-dimensional BSDE (Lemma 5 in the Appendix B.1.1), the unique equilibrium result holds in the team production model even when $U$ is discontinuous in $X_{T}$ or depends on the entire path $\left(X_{t}\right)_{0 \leq t \leq T}$.

\subsection{Hold-Up Problem}

In this subsection we study a class of hold-up problems that highlight a form of commitment power embedded in the gradually evolving state. In particular, we obtain a closed-form solution for the equilibrium strategy and show that a certain level of "cooperation" can be achieved, which is not possible under the static benchmark. This also illustrates the point that Nash equilibrium outcomes in the game with gradual adjustments can be very different from those under the static benchmark model even if the

\footnotetext{
${ }^{20}$ We thank an anonymous referee for suggesting this simulation experiment.
} 


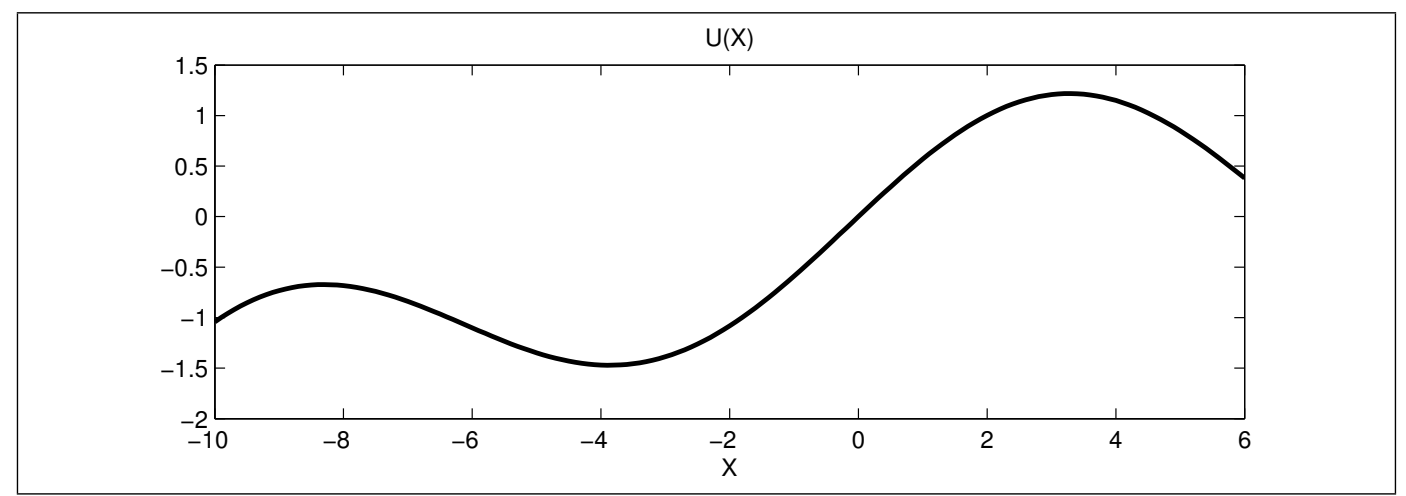

Figure 1: Terminal payoff function with multiple local maxima
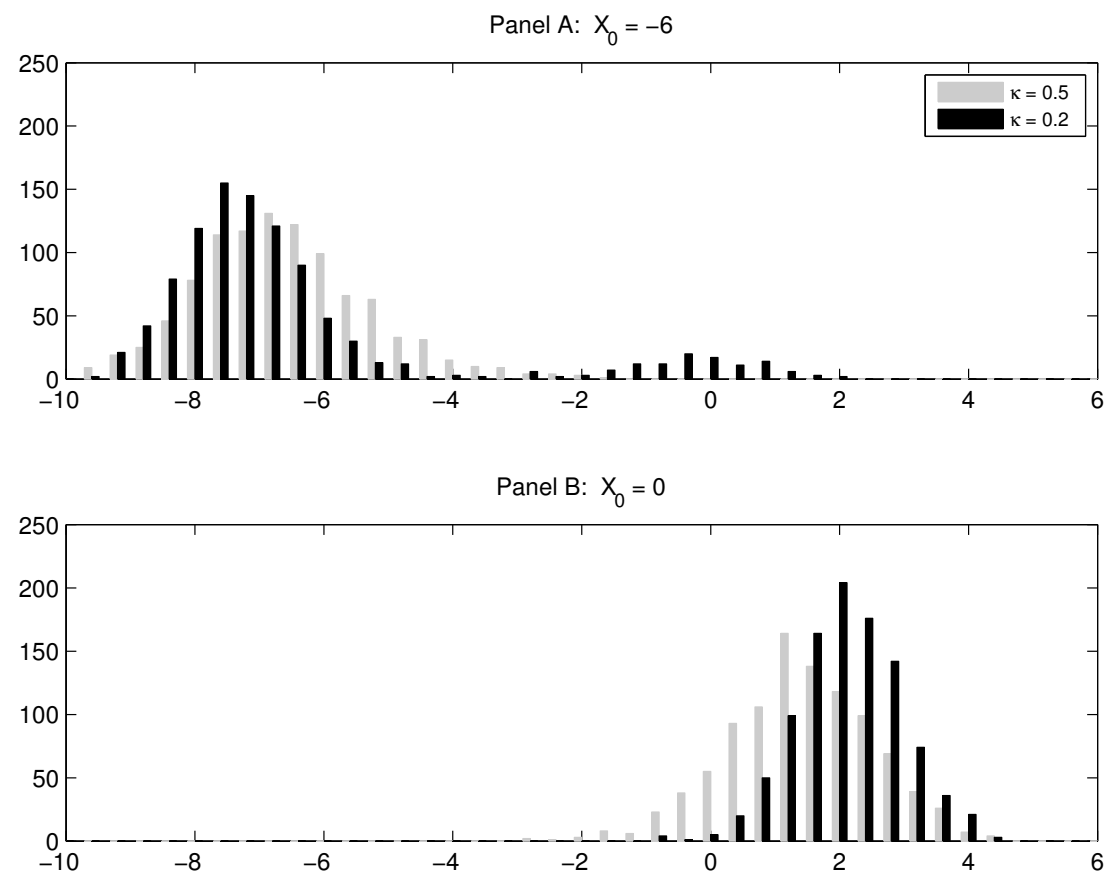

Panel C: $\mathrm{X}_{0}=-5.2$

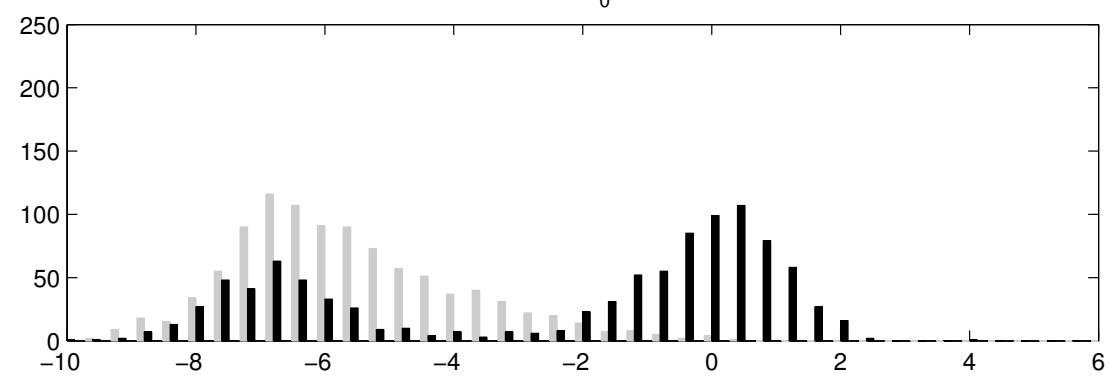

Figure 2: Simulated distributions of $X_{T}$ with different initial points $X_{0}$ and cost levels $\kappa$. In each panel, we fix $X_{0} \in\{-6,0,-5.2\}$ and compare the simulated distributions of $X_{T}$ with $\kappa=0.5$ (grey) and 0.2 (black). For each parameter set, we simulated $X_{T} 1000$ times and plotted them as a histogram. The other parameters are $\sigma=1, N=2$, and $T=1$. 
noise level is arbitrarily small.

Suppose that $d=n=2$, and there are a seller (player 1) and a buyer (player 2). Player $i$ controls his/her own one-dimensional state $d X_{t}^{i}=A_{t}^{i} d t+d Z_{t}^{i}$ given $X_{0}^{i}=0$. The seller chooses investment $A_{t}^{1}$ to improve the quality of a good $X_{t}^{1}$ by incurring a flow $\operatorname{cost} c^{1}\left(A_{t}^{1}\right)$. The buyer decides the amount of the purchase, $X_{t}^{2}$. His adjustment cost $c^{2}\left(A_{t}^{2}\right)$ captures the transaction costs of - or the frictions involved in loading - new goods, such as preparing the necessary facilities. The price per unit is exogenously fixed. The seller's terminal payoff $U^{1}$ is her revenue, and the buyer's terminal payoff $U^{2}$ is his utility of consumption. We make the following assumptions: (i) $\frac{\partial U^{1}}{\partial X^{2}}>0$, that is, the seller's benefit is increasing in the amount of the buyer's purchase; (ii) $\frac{\partial U^{1}}{\partial X^{1}}=0$, that is, the seller's revenue does not depend on the quality per se; (iii) $\frac{\partial^{2} U^{2}}{\partial X^{1} \partial X^{2}}>0$; and (iv) $c^{1}$ is minimized at 0 .

When the quality of a good is not contractible, incentivizing the seller to make a costly investment is a difficult problem in general. The seller and buyer could negotiate the price of the good after observing the final quality, but this approach may not be helpful when the buyer gains bargaining power ex post. In this subsection, we examine an alternative scheme in which the seller and buyer agree on a fixed price ex ante and the buyer gradually adjusts the scheduled amount of the purchase while observing the seller's quality investment thus far. In this setting, the seller has an incentive to choose a positive level of investment under the dynamic model, because it will encourage the buyer to purchase the good at a later time. ${ }^{21}$ This is in contrast to the static benchmark case (Section 2.2), where the seller chooses the dominant strategy, $A_{0}^{1}=0$.

To quantify the welfare gain, we focus on the following quadratic payoffs to obtain a closed-form solution:

$$
U^{1}(X)=X^{2}, \quad U^{2}(X)=X^{1} X^{2}, \quad c^{i}\left(A^{i}\right)=\frac{\bar{c}_{i}}{2}\left(A^{i}\right)^{2}
$$

for some $\bar{c}_{1}, \bar{c}_{2}>0$ with $\mathcal{A}^{i}=\mathbb{R}$. In Supplementary Appendix D, we show that a linear equilibrium in a general quadratic game is obtained by using a system of ordinary differential equations, which is independent of $\sigma$. Accordingly, we obtain the following

\footnotetext{
${ }^{21}$ The logic is similar to that of Bohren (2014), who studies an infinite-horizon model in which a long-lived seller is faced with a continuum of short-lived buyers at each moment in time. The seller has an incentive to make an investment, since the effect is persistent over time, which will convince prospective future buyers to make purchases. In our model, the buyer is not myopic, and his forward-looking incentive is captured by the cooperation term.
} 
result.

Proposition 3. Consider the hold-up problem with quadratic payoffs. Then there is a Nash equilibrium $A=\left(A^{i}\right)_{i=1,2}$ given by

$$
A_{t}^{1}=\underbrace{\frac{T-t}{\bar{c}_{1} \bar{c}_{2}}}_{\text {cooperation term }}, \quad A_{t}^{2}=\underbrace{\frac{(T-t)^{2}}{2 \bar{c}_{1}\left(\bar{c}_{2}\right)^{2}}}_{\text {cooperation term }}+\frac{X_{t}^{1}}{\bar{c}_{2}} .
$$

Moreover, the corresponding equilibrium payoffs are as follows:

$$
W_{0}^{1}(A)=\frac{T^{3}}{3 \bar{c}_{1}\left(\bar{c}_{2}\right)^{2}}, \quad W_{0}^{2}(A)=\frac{T^{2} \sigma^{2}}{4 \bar{c}_{2}}+\frac{3 T^{5}}{20\left(\bar{c}_{1}\right)^{2}\left(\bar{c}_{2}\right)^{3}}
$$

The basic reason for the welfare improvement is that the seller can acquire commitment power from the gradually evolving state, which encourages the seller's investment. Also, anticipating the positive investment incentive by the seller, the buyer has an additional incentive to increase the purchase amount. These effects are captured by the "cooperation terms" in the above formula, and disappear as time $t$ approaches the deadline $T .^{22}$

As an extreme form of a seller's commitment power, we can compute the Stackelberg equilibrium in the static benchmark game, where the seller chooses $A_{0}^{1}=\frac{T}{\bar{c}_{1} \bar{c}_{2}}$, and the buyer chooses $A_{0}^{2}=\frac{T}{\bar{c}_{1} \bar{c}_{2}}$. The seller's payoff is $\frac{T^{3}}{2 \bar{c}_{1}\left(\bar{c}_{2}\right)^{2}}$, which is always higher than the dynamic equilibrium payoff in Proposition 3. The buyer's payoff is $\frac{T^{5}}{2\left(\bar{c}_{1}\right)^{2}\left(\bar{c}_{2}\right)^{3}}$, which can be lower than the dynamic equilibrium payoff if $\sigma$ is high. This captures the value of action flexibility in a dynamic setting that is missing in the static case. The payoffs in the static benchmark case are not affected even if a noise term $Z_{T}$ affects the state variables.

\subsection{Dynamic Contest}

Next, we apply our framework to a contest model. ${ }^{23}$ In this class of games, players compete for a fixed prize, and the value of the prize does not depend on their effort. As an example, consider an internal labor market where colleagues compete for a promotion. The boss gives a promotion to the employee whose project receives the highest evaluation. In our model, each player chooses her effort level at each time to improve the quality of her project, and the boss evaluates performance based on the final quality of each player's project at deadline $T$.

\footnotetext{
${ }^{22}$ We note that the unbounded control ranges deviate from the original model formulation, and thus the equilibrium uniqueness is an open question.

${ }^{23}$ See Konrad (2009) for an extensive survey of this field.
} 
Consider the case of two players $(d=2)$. Player $i$ exerts costly effort $A_{t}^{i}$ at each time $t$ to improve the quality $X_{t}^{i}$ of her project, which follows $d X_{t}^{i}=A_{t}^{i} d t+d Z_{t}^{i}$ given the initial value $X_{0}^{i}$. We normalize the payoff for the winner of the prize to 1 . We suppose the winner is stochastically determined with the following additive noise:

$$
U^{i}(X)=\operatorname{Pr}\left[X^{i}+\theta^{i}>X^{j}+\theta^{j}\right]
$$

where $\theta^{i}$ is a random element that affects the contest designer's judgment. Hence the terminal payoff for player $i$ is increasing in the state difference $X_{T}^{i}-X_{T}^{j}$. By assuming that the distribution of $\theta:=\theta^{1}-\theta^{2}$ admits a bounded density, the terminal payoff function $U^{i}$ becomes Lipschitz continuous.

In the dynamic model, the players' equilibrium controls depend on their relative positions, which reflect their dynamic strategic incentives. To examine this point, we numerically simulated the equilibrium PDE using the symmetric quadratic cost funcitons $c^{i}(a)=\frac{\bar{c}}{2} a^{2}$ with $\mathcal{A}^{i}=\mathbb{R}$ and terminal payoffs with logit noise $U^{i}(X)=\frac{\exp \left[\gamma X^{i}\right]}{\exp \left[\gamma X^{1}\right]+\exp \left[\gamma X^{2}\right]}$, where $\gamma>0$. The numerical simulation results indicate that the leader (i.e., the player with the higher value of the state variable) generally exerts greater effort than the follower. The leader has an incentive to "discourage" the follower and get her to exert less effort.

Relative to the static benchmark case, we observe that the expected total payoff for each player is higher than in the dynamic setting (Figure 3). That is, the total cost of effort is lower in expectation. This is because the players tend to exert less effort under the dynamic model when the state difference becomes large (Figure 4).

Another observation from the simulations is that the equilibrium effort level $a^{i}(t, X)$ is monotone in $t$ (Figure 4). In particular, it is increasing in $t$ when competition is fierce, that is, when $X^{1} \approx X^{2}$. To understand this, note that the benefit of exerting effort in such a situation is greater when $t$ is closer to the deadline, because the magnitude of the remaining Brownian noise is smaller. On the other hand, consider the case where $\left|X_{t}^{1}-X_{t}^{2}\right|$ is large. Then the winner of the contest is essentially already determined if $t$ is close to the deadline, hence the players tend to stop choosing high effort levels, and this causes $a^{i}$ to be decreasing in $t .^{25}$

\footnotetext{
${ }^{24}$ See Appendix A. We used the unbounded control range in the simulation for simplicity.

${ }^{25}$ The simulations suggest that $a^{i}$ tends to be increasing in $t$, especially when $\gamma$ is small. To interpret this, note that in more random contests (smaller $\gamma$ ), raising the level of effort has a greater marginal effect on the winning probability. Therefore, the players become competitive even when the difference $\left|X^{1}-X^{2}\right|$ is large.
} 
Harris and Vickers (1987) study a related model of continuous-time contest games, called the tug-of-war model. ${ }^{26}$ In this model, the players exert effort to influence the drift of their state variables, as in the current paper. As in our model, in each state the leader always exerts more effort than the follower. In contrast to our model, there is no fixed deadline; one of the players wins, and the game ends if the difference between the values of the state variables reaches some pre-established value. Harris and Vickers focus on time-stationary strategies, while our model allows us to investigate the effects of the length of time remaining (before the deadline) on equilibrium behavior.

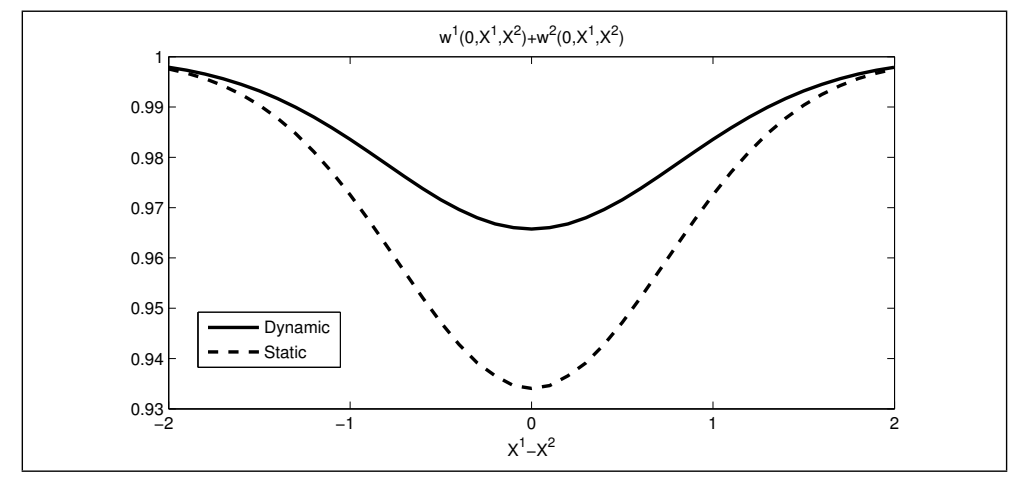

Figure 3: Expected total payoff of the players under different initial state differences $X_{0}^{1}-X_{0}^{2}$. The total payoff under the dynamic model (solid line) exceeds that under the static model (dashed line) for each initial state. The other parameters are $\sigma=1$ and $\gamma=1$.

\section{Conclusion}

This study examines a class of games that embeds one-shot strategic interactions into a richer continuous-time setting. The equilibrium uniqueness result holds even if the adjustment costs and noise are arbitrarily small-and, more interestingly, even if the corresponding one-shot model generates multiple equilibria. The result suggests that, as long as our model seems to be a more realistic description than the one-shot modeling, the multiple equilibrium problem in static games could be attributed to over-simplification that abstracts away certain dynamic aspects. We also identify certain situations in which the model with gradual adjustment predicts higher efficiency than the one-shot setting.

\footnotetext{
${ }^{26}$ Budd, Harris, and Vickers (1993) analyze a more general model. Cao (2014) also studies a model with asymmetric players and the effect of a handicapping policy. Yildirim (2005) examines a discrete-time contest model with multiple periods. Notable differences from our result are as follows: (i) There are multiple equilibria when the players are asymmetric, and (ii) the total effort is typically at least as great as that in the one-shot model. Seel and Strack (Forthcoming) consider a continuous-time model in which players solve the optimal stopping problem with a private observation of the own state variables.
} 


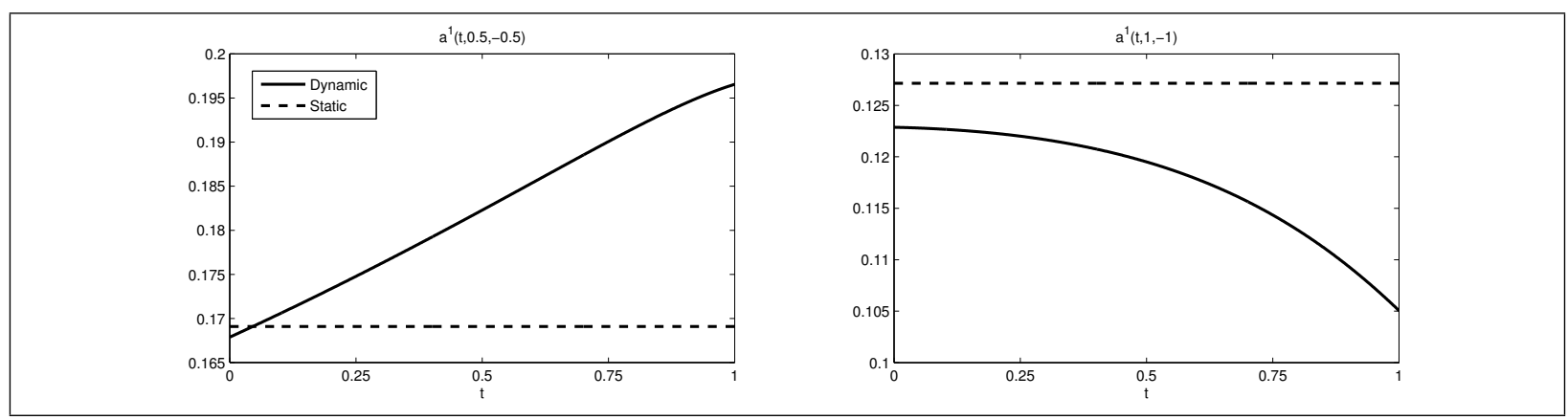

Figure 4: Time dynamics of player 1's optimal control policy in the dynamic contest game when player 1 is the leader. The solid lines are equilibrium strategies under the dynamic model: The effort level $a^{1}(t, X)$ is increasing in $t$ when $X_{t}^{1}$ and $X_{t}^{2}$ are close (left panel, with $X_{t}^{1}-X_{t}^{2}=1$ ) but decreasing in $t$ otherwise (right panel, with $X_{t}^{1}-X_{t}^{2}=2$ ). The dashed lines are the equilibrium effort levels under the static benchmark for different initial states. The other parameters are $\sigma=1$ and $\gamma=1$.

These results might be useful for understanding how timing of agents' decisions affects welfare, which is relevant in designing policies and institutions.

In general, we believe that the BSDE-based approach provides powerful tools that are applicable to broad classes of continuous-time games in which the standard HJB equations cannot be used. For example, the theory of BSDEs in settings with an infinite horizon and/or general Levy processes could be useful in other classes of economic models beyond the finite-horizon model with Brownian noise in this paper.

In Iijima and Kasahara (2015), we show the uniqueness of an open-loop equilibrium, in which players observe only the $Z_{t}$ process. Moreover, if there exists a potential function, the unique equilibrium maximizes the potential in the small friction limit, which serves as an equilibrium selection result. ${ }^{27}$

\footnotetext{
${ }^{27}$ The theory of global games (Carlsson and van Damme, 1993) is another equilibrium selection approach that enriches a complete information game by introducing uncertainty about economic fundamentals, which leads to equilibrium uniqueness. In contrast to our approach, the presence of private information is essential for the equilibrium uniqueness result in global games. Note that, while global games with strategic substitutes can have multiple equilibria, even such a class of games have a unique equilibrium in our framework.
} 


\section{Appendix}

\section{A PDE Characterization}

In this section, we derive a PDE characterization of the unique equilibrium, where the continuation value solves a system of PDEs. Our intention is to facilitate numerical calculation of the unique equilibrium, as it is often useful to be able to compute an equilibrium numerically in applications. ${ }^{28}$ In order to derive a PDE characterization, we impose the following conditions.

\section{Assumption 3.}

1. $c^{i}$ is $C^{2}$ and bounded. In addition, $c^{i}$ is strictly convex in $A^{i}$, and $\frac{\partial c^{i}\left(A^{i}, X\right)}{\partial A^{i}} \rightarrow \infty$ as $A^{i} \rightarrow \operatorname{bd}\left(\mathcal{A}^{i}\right)$.

2. $U^{i}$ is $C^{3}$ and has bounded derivatives.

Assumption 3.1 ensures that player $i$ 's optimal action is in the interior of $\mathcal{A}^{i}$ and $f^{i}$ is continuously differentiable. Note that Assumption 3 is not consistent with Assumption 2. We now introduce a restriction on the class of equilibria.

Definition 1. A bounded sensitivity equilibrium is a Nash equilibrium $A=\left(A^{i}\right)_{i \in N}$ such that $\left|\beta_{t}(A)\right|$ is bounded, that is, there exists $M>0$ such that $\left|\beta_{t}^{i}(A)\right|<M$ for any $i$ and for almost all $t$ almost surely.

Proposition 4. Under Assumptions 1 and 3, if $\mu_{0}$ and $\mu_{i}$ for each $i \in N$ are bounded and continuously differentiable, then there exists a unique bounded sensitivity equilibrium $A$. Its continuation value take the form $W_{t}^{i}(A)=w^{i}\left(t, X_{t}\right)$, where $w=\left(w^{i}\right)_{i \in N}$ is a unique solution to the following system of PDEs:

$0=\frac{\partial w^{i}(t, x)}{\partial t}+\frac{1}{2} \sum_{l, m=1}^{d} \sum_{k=1}^{d} \sigma_{l k} \sigma_{m k} \frac{\partial^{2} w^{i}(t, x)}{\partial x^{l} \partial x^{m}}+\left(\sum_{j \in N} \mu_{j} a^{j}+\mu_{0}(x)\right) \cdot \nabla_{x} w^{i}(t, x)-c^{i}\left(a^{i}, x\right)$,

where $a^{i}(t, x)=f^{i}\left(\nabla_{x} w^{i}(t, x), x\right)$ for all $t, x$ with a terminal condition $w^{i}(T, x)=U^{i}(x)$.

The equilibrium strategy takes the form $A_{t}^{i}=f^{i}\left(\mu_{i}\left(X_{t}\right) \cdot \nabla_{x} w^{i}\left(t, X_{t}\right), X_{t}\right)$.

\footnotetext{
${ }^{28}$ Numerical calculation of Markovian BSDEs is an active research area, and we note that many papers that use Monte Carlo simulations as well (Gobet, Lemor, and Warin, 2005).
} 
Here we give only a sketch of the proof; the details are provided in Supplementary Appendix E.2. First, we construct a particular bounded sensitivity equilibrium by solving the HJB equations that lead to the PDEs (8). We rely on the theory of uniformly parabolic PDEs (Ladyzenskaja, Solonnikov, and Ural'ceva, 1995) to prove the existence of a unique solution to the system of PDEs. Assumption 3 is used in this step to ensure the continuous differentiability of $f^{i}$. Then we show that the constructed strategy profile is a unique bounded sensitivity equilibrium, by truncating the original BSDEs and applying the uniqueness result for uniform Lipschitz BSDEs (Pardoux and Peng, 1990). The bounded sensitivity assumption is used in justifying the truncation step. It is an open question whether Nash equilibria with unbounded sensitivity exist.

\section{B Proofs of Main Results}

\section{B.1 Preliminaries}

This section introduces results from the BSDE literature that we rely on for the proofs.

\section{B.1.1 BSDEs}

In this subsection, we define BSDEs and introduce relevant results. ${ }^{29}$

Fix any invertible $d \times d$ matrix $\tilde{\Sigma}$ throughout this subsection. Let $X=\left\{X_{t}\right\}_{0 \leq t \leq T}$ be a standard $d$-dimensional Brownian motion on a filtered probability space $(\Omega, \mathcal{F}, \mathbb{P})$, where $\mathcal{F}=\left\{\mathcal{F}_{t}\right\}_{0 \leq t \leq T}$ is the augmented filtration generated by $X$, and $T$ is a fixed finite horizon. We denote by $\mathbb{S}^{2}$ the set of $\mathbb{R}^{n}$-valued progressively measurable processes $W$ over $[0, T]$ such that

$$
\mathbb{E}\left[\sup _{0 \leq t \leq T}\left|W_{t}\right|^{2}\right]<\infty,
$$

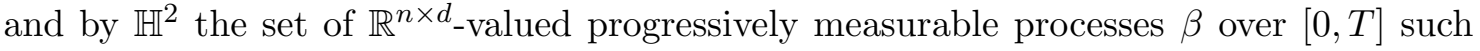
that

$$
\mathbb{E}\left[\int_{0}^{T}\left|\beta_{t}\right|^{2} d t\right]<\infty
$$

We call $g: \Omega \times[0, T] \times \mathbb{R}^{n} \times \mathbb{R}^{n \times d} \rightarrow \mathbb{R}^{n}$ a driver if the following conditions hold:

- $g(\omega, t, w, b)$, written $g(t, w, b)$ for simplicity, is progressively measurable for all $w \in$

\footnotetext{
${ }^{29}$ See, for example, Pham (2010). Note that the notation $(W, \beta)$ used here corresponds to $(Y, Z)$ in the standard notation.
} 


$$
\begin{gathered}
\mathbb{R}^{n} \text { and } b \in \mathbb{R}^{n \times d} \\
\text { - } g(t, 0,0) \in \mathbb{S}^{2}(0, T)^{n}
\end{gathered}
$$

We call $\xi$ a terminal condition if it is an $\mathbb{R}^{n}$-valued random variable such that $\mathbb{E}\left[|\xi|^{2}\right]<\infty$.

Definition 2. Let $\xi$ be a terminal condition, and let $g$ be a driver. A BSDE is a stochastic system of equations defined by ${ }^{30}$

$$
W_{t}=\xi+\int_{t}^{T} g\left(s, W_{s}, \beta_{s}\right) d s-\int_{t}^{T} \beta_{s} \cdot \tilde{\Sigma} d X_{s}, \quad 0 \leq t \leq T
$$

A solution to a BSDE is a pair $(W, \beta) \in \mathbb{S}^{2} \times \mathbb{H}^{2}$ that satisfies the above system of equations. A BSDE is said to be one-dimensional if $n=1$.

We often write the above BSDEs in differential form as

$$
d W_{t}=-g\left(t, W_{t}, \beta_{t}\right) d t+\beta_{t} \cdot \tilde{\Sigma} d X_{t}, \quad W_{T}=\xi
$$

The following result is due to Pardoux and Peng (1990).

Lemma 3 (Lipschitz BSDE). Let $\xi$ be a terminal condition, and let $g$ be a driver that satisfies the uniform Lipschitz condition. That is, there exists a constant $C_{g}$ such that

$$
|g(t, w, b)-g(t, \tilde{w}, \tilde{b})| \leq C_{g}(|w-\tilde{w}|+|b-\tilde{b}|), \quad \text { for all } w, \tilde{w}, b, \tilde{b}, \quad d t \otimes d \mathbb{P} \text { a.e. }
$$

Then there exists a unique solution $(W, \beta)$ of the BSDE.

A driver $g$ satisfies the quadratic growth condition if there is a constant $C>0$ such that

$$
\begin{gathered}
|g(t, w, b)| \leq C\left(1+|w|+|b|^{2}\right), \\
|g(t, w, b)-g(t, \tilde{w}, \tilde{b})| \leq C(|w-\tilde{w}|+(|w|+|b|+|\tilde{w}|+|\tilde{b}|)|b-\tilde{b}|)
\end{gathered}
$$

for all $\omega \in \Omega, t \in[0, T], w, \tilde{w} \in \mathbb{R}^{n}$, and $b, \tilde{b} \in \mathbb{R}^{n \times d}$.

The following results for one-dimensional BSDEs are due to Kobylanski (2000).

Lemma 4 (Comparison Theorem). Consider a pair of one-dimensional BSDEs that are associated with drivers and terminal conditions $(\xi, g)$ and $(\tilde{\xi}, \tilde{g})$ that satisfy the quadratic

\footnotetext{
${ }^{30}$ In the standard notation, $\tilde{\Sigma}$ is usually an identity matrix. We chose to include this additional term, which does not change any of the results in this section, in order to make it easier to see their applicability to our model.
} 
growth condition. Let $(W, \beta)$ and $(\tilde{W}, \tilde{\beta})$ be the corresponding solutions. If $\xi \geq \tilde{\xi}$ almost surely and $g\left(t, W_{t}, \beta_{t}\right) \geq \tilde{g}\left(t, W_{t}, \beta_{t}\right)$ for almost all $t$ almost surely, then $W_{t} \geq \tilde{W}_{t}$ for all $t$ almost surely. If, in addition, $g\left(t, W_{t}, \beta_{t}\right)>\tilde{g}\left(t, W_{t}, \beta_{t}\right)$ holds for a strictly positive measure under $d t \otimes d \mathbb{P}$, then $W_{0}>\tilde{W}_{0}$.

Lemma 5 (One-dimensional Quadratic BSDE). Consider a one-dimensional BSDE in which $\xi$ is a terminal condition and $g$ is a driver that satisfies the quadratic growth condition. Then there exists a unique solution $(W, \beta)$ of the BSDE.

\section{B.1.2 FBSDE}

Fix a probability space $(\Omega, \mathcal{G}, \mathbb{Q})$ in which $\left\{\mathcal{G}_{t}\right\}_{0 \leq t \leq T}$ satisfies the usual conditions and $\left(Z_{t}\right)_{0 \leq t \leq T}$ is a standard $d$-dimensional $\mathcal{G}_{t}$-Brownian motion. Note that $\mathcal{G}_{t}$ can be larger than the filtration generated by the Brownian motion $Z_{t}$. A (Markovian) forward backward stochastic differential equation (FBSDE) is a stochastic system of equations that takes the form

$$
\begin{aligned}
& d X_{t}=F\left(t, X_{t}, W_{t}, \beta_{t}\right) d t+\tilde{\Sigma} d Z_{t}, \quad X_{0}=x_{0} \\
& d W_{t}=G\left(t, X_{t}, W_{t}, \beta_{t}\right) d t+\beta_{t} \cdot d Z_{t}, \quad W_{T}=U\left(X_{T}\right)
\end{aligned}
$$

for some $F:[0, T] \times \mathbb{R}^{d} \times \mathbb{R}^{n} \times \mathbb{R}^{n \times d} \rightarrow \mathbb{R}^{n}, G:[0, T] \times \mathbb{R}^{d} \times \mathbb{R}^{n} \times \mathbb{R}^{n \times d} \rightarrow \mathbb{R}^{d}, U: \mathbb{R}^{d} \rightarrow \mathbb{R}^{n}$, $x_{0} \in \mathbb{R}^{d}$, and invertible $\tilde{\Sigma} \in \mathbb{R}^{d \times d}$.

A solution $(X, W, \beta)$ to an FBSDE is an $\mathbb{R}^{d} \times \mathbb{R}^{n} \times \mathbb{R}^{n \times d}$-valued $\left\{\mathcal{G}_{t}\right\}$-progressively measurable process that satisfies $(9)$ and

$$
\mathbb{E}\left[\int_{0}^{T}\left(\left|X_{t}\right|^{2}+\left|W_{t}\right|^{2}+\left|\beta_{t}\right|^{2}\right) d t\right]<\infty
$$

The following result is due to Delarue $(2002) \cdot{ }^{31}$

Lemma 6 (Non-degenerate FBSDE). Consider the FBSDE (9). Assume that there exists a constant $K>0$ such that the following conditions are satisfied (for all $t, x, x^{\prime}, w, w^{\prime}, b, b^{\prime}$ ):

\footnotetext{
${ }^{31}$ Delarue (2002) uses a more general formulation that, in particular, allows the matrix $\tilde{\Sigma}$ to depend on $t$ and $X$. He requires the condition called uniform parabolicity: There exist $\nu, \nu^{\prime}>0$ such that $\forall \xi \in \mathbb{R}^{d}, \nu \leq$ $\frac{1}{|\xi|^{2}} \xi^{\prime}\left(\tilde{\Sigma}(t, X) \tilde{\Sigma}^{\prime}(t, X)\right) \xi \leq \nu^{\prime}$ for all $t, X$. To see that this condition is satisfied in the current framework, first note that $\tilde{\Sigma} \tilde{\Sigma}^{\prime}$ has $d$ real positive eigenvalues $\left(\lambda_{1}, \cdots, \lambda_{d}\right)$, because $\tilde{\Sigma}$ is invertible, which guarantees that $\tilde{\Sigma} \tilde{\Sigma}^{\prime}$ is positive definite. By the standard results in linear algebra, $0<\left(\min _{k} \lambda_{k}\right)|\xi|^{2} \leq \xi^{\prime}\left(\tilde{\Sigma} \tilde{\Sigma}^{\prime}\right) \xi \leq\left(\max _{k} \lambda_{k}\right)|\xi|^{2}$ for any non-zero $\xi \in \mathbb{R}^{d}$.
} 
1. $F$ is continuous in $x$ and satisfies

$$
\begin{aligned}
& \left|F(t, x, w, b)-F\left(t, x, w^{\prime}, b^{\prime}\right)\right| \leq K\left(\left|w-w^{\prime}\right|+\left|b-b^{\prime}\right|\right) \\
& \left(x-x^{\prime}\right) \cdot\left(F(t, x, w, b)-F\left(t, x^{\prime}, w, b\right)\right) \leq K\left|x-x^{\prime}\right|^{2} \\
& |F(t, x, w, b)| \leq K(1+|x|+|w|+|b|)
\end{aligned}
$$

2. $G$ is continuous in $w$ and satisfies

$$
\begin{aligned}
& \left|G(t, x, w, b)-G\left(t, x^{\prime}, w, b^{\prime}\right)\right| \leq K\left(\left|x-x^{\prime}\right|+\left|b-b^{\prime}\right|\right) \\
& \left(w-w^{\prime}\right) \cdot\left(G(t, x, w, b)-G\left(t, x, w^{\prime}, b\right)\right) \leq K\left|w-w^{\prime}\right|^{2} \\
& |G(t, x, w, b)| \leq K(1+|x|+|w|+|b|)
\end{aligned}
$$

3. $U$ is Lipschitz continuous and bounded, that is, it satisfies

$$
\begin{aligned}
& \left|U(x)-U\left(x^{\prime}\right)\right| \leq K\left(\left|x-x^{\prime}\right|\right) \\
& |U(x)| \leq K .
\end{aligned}
$$

Then there exists a unique solution $(X, W, \beta)$ of the FBSDE. The solution takes the form $W_{t}=w\left(t, X_{t}\right), \beta_{t}=b\left(t, X_{t}\right)$ for some function $w:[0, T] \times \mathbb{R}^{d} \rightarrow \mathbb{R}^{n}$ that is uniformly Lipschitz continuous in $x \in \mathbb{R}^{d}$, and some function $b:[0, T] \times \mathbb{R}^{d} \rightarrow \mathbb{R}^{d \times n}$.

\section{B.2 Proof of Lemma 1}

We note that the model is constructed from a canonical probability space $(\Omega, \mathcal{F}, \mathbb{P})$ where there is no drift in $X$ and changes in the probability measure depend on $A=\left(A^{i}\right)_{i \in N}$. We denote this new probability space by $\left(\Omega, \mathcal{F}, \mathbb{P}^{A} ; \mathcal{F}^{X}\right)$, which has been augmented with the filtration generated by $X$, and the the corresponding expectation operator by $\mathbb{E}^{A}$. This construction is standard and (often implicitly) used by most continuous-time models that feature imperfect public monitoring. ${ }^{32}$

We begin with the following preliminary lemma.

Lemma 7. Fix a strategy profile $A=\left(A^{i}\right)_{i \in N}$. Then $W_{0}^{i}(A) \geq W_{0}^{i}\left(\hat{A}^{i},\left(A^{j}\right)_{j \neq i}\right)$ for any strategy $\hat{A}^{i}$ of player $i$ if and only if $A_{t}^{i}=f^{i}\left(\beta_{t}(A), X_{t}\right)$ for almost all $t \in[0, T]$ almost

\footnotetext{
${ }^{32}$ Chapter 5 of Cvitanic and Zhang (2012) discusses the technical details more extensively in the context of dynamic contracts.
} 
surely.

Proof. Fix any strategy profile $A=\left(A^{i}\right)_{i \in N}$. As discussed in Section 3.1, player $i$ 's continuation payoff under $A$ takes the form

$$
\begin{aligned}
W_{t}^{i}(A) & =\mathbb{E}^{A}\left[U^{i}\left(X_{T}\right)-\int_{t}^{T} c^{i}\left(A_{s}^{i}, X_{s}\right) d s \mid \mathcal{F}_{t}^{X}\right] \\
& =U^{i}\left(X_{T}\right)-\int_{t}^{T} c^{i}\left(A_{s}^{i}, X_{s}\right) d s-\int_{t}^{T} \beta_{s}^{i}(A) \cdot d Z_{s}(A) \\
& =U^{i}\left(X_{T}\right)-\int_{t}^{T}\left(c^{i}\left(A_{s}^{i}, X_{s}\right)-\beta_{s}^{i}(A) \cdot \Sigma^{-1}\left(\sum_{j \in N} \mu_{j} A_{s}^{j}+\mu_{j}\left(X_{s}\right)\right)\right) d s-\int_{t}^{T} \beta_{s}^{i}(A) \cdot \Sigma^{-1} d X_{s}
\end{aligned}
$$

with $W_{T}^{i}(A)=U^{i}\left(X_{T}\right)$. The second equality follows by applying the extended martingale representation theorem to the conditional expectation of the total payoff (see for example Chapter 5 and Lemma 10.4.6 in Cvitanic and Zhang (2012)), and the third equality uses $d X_{t}=\left(\sum_{j} \mu_{j} A_{t}^{j}+\mu_{0}\left(X_{t}\right)\right) d t+\Sigma d Z_{t}$.

The pair $\left(W^{i}(A), \beta^{i}(A)\right)$ is a solution to the one-dimensional BSDE (under the original probability space) with driver $-c^{i}\left(A_{t}^{i}, X_{t}\right)+\beta^{i} \cdot\left(\Sigma^{-1} \sum_{j \in N} \mu_{j} A_{t}^{j}+\mu_{0}\left(X_{t}\right)\right)$ and terminal condition $U_{T}^{i}\left(X_{T}\right)$. Note that we treat $\left(A^{j}\right)_{j \neq i}$ as exogenous processes in this BSDE formulation.

Take another strategy profile $\tilde{A}=\left(\tilde{A}^{j}\right)_{j \in N}$ in which player $i$ uses strategy $\tilde{A}_{t}^{i}=$ $f^{i}\left(\beta_{t}(A), X_{t}\right)$ and where $\tilde{A}^{j}=A^{j}$ for every $j \neq i$. Using the same argument as above, player $i$ 's continuation value takes the form

$W_{t}^{i}(\tilde{A})=U^{i}\left(X_{T}\right)-\int_{t}^{T}\left(c^{i}\left(\tilde{A}_{s}^{i}, X_{s}\right)-\beta_{s}^{i}(\tilde{A}) \cdot \Sigma^{-1}\left(\sum_{j} \mu_{j} \tilde{A}_{s}^{j}+\mu_{0}\left(X_{s}\right)\right)\right) d s-\int_{t}^{T} \beta_{s}^{i}(\tilde{A}) \cdot \Sigma^{-1} d X_{s}$

with $W_{T}^{i}(\tilde{A})=U^{i}\left(X_{T}\right)$. Likewise, $\left(W^{i}(\tilde{A}), \beta^{i}(\tilde{A})\right)$ is a solution to the one-dimensional BSDE with driver $-c^{i}\left(\tilde{A}_{t}^{i}, X_{t}\right)+\beta^{i} \cdot \Sigma^{-1}\left(\sum_{j} \mu_{j} \tilde{A}_{t}^{j}+\mu_{0}\left(X_{t}\right)\right)$ and terminal condition $U^{i}\left(X_{T}\right)$. Note that the driver term satisfies the quadratic growth condition, because $f^{i}$ is bounded and Lipschitz continuous (Lemma 8). By the comparison theorem (Lemma 4), $W_{0}^{i}(\tilde{A}) \geq$ $W_{0}^{i}(A)$ holds. This proves the "if" direction.

To prove the "only if" direction, consider the case in which $A_{t}^{i} \neq f^{i}\left(\beta_{t}, X_{t}\right)$ for some strictly positive measure $d t \otimes d \mathbb{P}$. Then by the strict inequality part of the comparison theorem (Lemma 4), $W_{0}^{i}(\tilde{A})>W_{0}^{i}(A)$. 
Proof of Lemma 1. To show the "if" direction, take any strategy profile $A=\left(A^{i}\right)_{i \in N}$ under which conditions (1), (3), (4), and (5) hold for some $\mathcal{F}^{X}$-progressively measurable processes $(W, \beta)$ such that $\mathbb{E}\left[\sup _{0 \leq t \leq T}\left|W_{t}\right|^{2}\right], E\left[\int_{0}^{T}\left|\beta_{t}\right|^{2} d t\right]<\infty$. As we have seen in the proof of Lemma 7 , for player $i$, the pair of processes $\left(W^{i}(A), \beta^{i}(A)\right)$ is a unique solution to one-dimensional BSDE with driver $-c^{i}\left(A_{t}^{i}, X_{t}\right)+\beta^{i} \cdot\left(\Sigma^{-1} \sum_{j \in N} \mu_{j} A_{t}^{j}+\mu_{0}\left(X_{t}\right)\right)$ and terminal condition $U_{T}^{i}\left(X_{T}\right)$. Thus $W_{t}^{i}=W_{t}^{i}(A)$ and $\beta_{t}^{i}=\beta_{t}^{i}(A)$ (for almost all $t$ almost surely). By Lemma 7 and the incentive compatibility condition (5), each player maximizes her expected total payoff. Thus $A$ is a Nash equilibrium.

To show the "only if" direction, take any Nash equilibrium $A=\left(A^{i}\right)_{i \in N}$. Then the incentive compatibility condition (5) holds by Lemma 7 . The remaining conditions follow immediately by setting $W_{t}^{i}=W_{t}^{i}(A)$ and $\beta_{t}^{i}=\beta_{t}^{i}(A)$.

\section{B.3 Proof of Theorem 1}

Lemma 8. There is $C_{f}>0$ such that $\left|f^{i}(\beta, X)-f^{i}(\tilde{\beta}, \tilde{X})\right| \leq C_{f}\left(\left|\beta^{i}-\tilde{\beta}^{i}\right|+|X-\tilde{X}|\right)$ for all $\beta, \tilde{\beta}, X, \tilde{X}$.

Proof. Denote the control range by $\mathcal{A}^{i}=\left[\underline{A}^{i}, \bar{A}^{i}\right]$. Since $\beta^{i} \cdot \Sigma^{-1} \mu_{i} \alpha^{i}-c^{i}\left(\alpha^{i}, X\right)$ is strictly concave in $\alpha^{i}$, its unique maximizer $A^{i}$ is continuous in $\beta$ and $X$ and satisfies the firstorder condition $\beta^{i} \cdot \Sigma^{-1} \mu_{i}=\frac{\partial c^{i}\left(A^{i}, X\right)}{\partial A^{i}}$ in the case of $A^{i} \in\left(\underline{A}^{i}, \bar{A}^{i}\right)$. Furthermore, this maximizer takes the form

$$
f^{i}(\beta, X)= \begin{cases}\bar{A}^{i} & \text { if } \beta^{i} \cdot \Sigma^{-1} \mu_{i} \geq \frac{\partial c^{i}\left(\bar{A}^{i}, X\right)}{\partial A^{i}} \\ g^{i}(\beta, X) & \text { if } \beta^{i} \cdot \Sigma^{-1} \mu_{i} \in\left(\frac{\partial c^{i}\left(\underline{A}^{i}, X\right)}{\partial A^{i}}, \frac{\partial c^{i}\left(\bar{A}^{i}, X\right)}{\partial A^{i}}\right) \\ \underline{A}^{i} & \text { if } \beta^{i} \cdot \Sigma^{-1} \mu_{i} \leq \frac{\partial c^{i}\left(\underline{A}^{i}, X\right)}{\partial A^{i}}\end{cases}
$$

where $g^{i}(\beta, X)$ is the unique value $A^{i} \in \mathcal{A}^{i}$ that is defined by the equality $\frac{\partial c^{i}\left(A^{i}, X\right)}{\partial A^{i}}-\beta^{i}$. $\Sigma^{-1} \mu_{i}=0$. Note that this value is uniquely determined, since $c^{i}$ is strictly convex in $A^{i}$. By the implicit function theorem, for $k=1, \ldots, d$, he first-order partial derivatives of $g^{i}$ satisfy

$$
\left|\frac{\partial g^{i}}{\partial X^{k}}\right|=\left|\frac{\frac{\partial^{2} c^{i}}{\partial A^{i} \partial X^{k}}}{\frac{\partial^{2} c^{i}}{\left(\partial A^{i}\right)^{2}}}\right|, \quad\left|\frac{\partial g^{i}}{\partial \beta^{i k}}\right|=\left|\frac{e^{k} \cdot \Sigma^{-1} \mu_{i}}{\frac{\partial^{2} c^{i}}{\left(\partial A^{i}\right)^{2}}}\right|,
$$

where $\beta^{i k}$ denotes the $k$ th element of the vector $\beta^{i} \in \mathbb{R}^{d}$, and $e_{k}$ is the $k$ th unit vector in $\mathbb{R}^{d}$. Since we can choose constants $M, M^{\prime}>0$ uniformly in $(\beta, X)$ such that $\left|\frac{\partial^{2} c^{i}}{\left(\partial A^{i}\right)^{2}}\right|>M$ 
and $\left|\frac{\partial^{2} c^{i}}{\partial A^{i} \partial X^{k}}\right|<M^{\prime}$ by Assumption 2.1, this establishes the Lemma.

Proof of Theorem 1. Take any solution $(W, \beta)$ of BSDE (6) it one exists. This is a solution to an FBSDE of the form

$$
\begin{aligned}
& d W_{t}^{i}=c^{i}\left(f^{i}\left(\beta_{t}, X_{t}\right)\right) d t+\beta_{t}^{i} \cdot d Z_{t}, \quad W_{T}^{i}=U^{i}\left(X_{T}\right), \quad i \in N \\
& d X_{t}=\left(\sum_{i} \mu_{i} f^{i}\left(\beta_{t}, X_{t}\right)+\mu_{0}\left(X_{t}\right)\right) d t+\Sigma d Z_{t}
\end{aligned}
$$

under the probability space $(\Omega, \mathcal{G}, \mathbb{Q})$ in which $\mathcal{G}=\mathcal{F}^{X}$ and the $Z_{t}$-process is a $d$ dimensional Brownian motion. The probability measure $\mathbb{Q}$ is constructed from the original canonical probability space $\left(\Omega, \mathcal{F}^{X}, \mathbb{P}\right)$ by the Girsanov theorem, which ensures $\mathcal{F}^{Z} \subseteq \mathcal{F}^{X}$.

We apply Lemma 6 to show that a unique solution $(X, W, \beta)$ to FBSDE (10) exists. To check condition 1 in Lemma 6, first note that the drift term in the expression for the $X_{t}$ process, namely $\sum_{i} \mu_{i} f^{i}\left(\beta, X_{t}\right)+\mu_{0}\left(X_{t}\right)$, is Lipschitz continuous in $\beta_{t}$ by Lemma 8 , which ensures the first inequality. The second inequality follows by the Cauchy-Schwartz inequality and the Lipschitz continuity of $\sum_{i} \mu_{i} f^{i}\left(\beta_{t}, X_{t}\right)+\mu_{0}\left(X_{t}\right)$ in $X$ (Lemma 8), and the boundedness of $\mu_{0}$ and $\mathcal{A}^{i}$ for each $i$ ensures the third inequality. To check condition 2 , note that the drift term in the expression for the $W_{t}^{i}$ process, namely $c^{i}\left(f^{i}(\beta, X), X\right)$, is Lipschitz continuous in $(X, \beta)$ by Lemma 8 and the assumptions on $c^{i}$. This term is independent of $W$, which ensures the second inequality. The third inequality follows from the boundedness of $c^{i}$ for each $i$. Condition 3 is satisfied by Assumption 2.2.

Thus if there is another solution $\left(W^{\prime}, \beta^{\prime}\right)$ to BSDE (6), this induces another solution $\left(X^{\prime}, W^{\prime}, \beta^{\prime}\right)$ to FBSDE (10) that coincides with the solution $(X, W, \beta)$. Thus $\left(W_{t}, \beta_{t}\right)=$ $\left(W_{t}^{\prime}, \beta_{t}^{\prime}\right)$ for almost all $t$ almost surely. By Theorem 2.1 of Cheridito and Nam (2015), the process $(W, \beta)$ is a solution to $\operatorname{BSDE}(6)$. This shows existence of a unique Nash equilibrium by Lemma 1.

Finally, by Lemma 6 , the unique solution $(X, W, \beta)$ to the FBSDE with $\mathcal{G}=\mathcal{F}^{Z}$ takes the form $W_{t}=w\left(t, X_{t}\right), \beta_{t}=b\left(t, X_{t}\right)$ for some function $w:[0, T] \times \mathbb{R}^{d} \rightarrow \mathbb{R}^{n}$ that is uniformly Lipschitz continuous in $x \in \mathbb{R}^{d}$ and some function $b:[0, T] \times \mathbb{R}^{d} \rightarrow \mathbb{R}^{d \times n}$. 


\section{B.4 Details for Section 3.2}

The continuation payoff for player $i$ at time $t=T-\Delta$ can be written as

$$
\begin{aligned}
W_{t}^{i, \Delta} & =-c^{i}\left(A_{t}^{i}, X_{t}\right) \Delta+\mathbb{E}\left[U^{i}\left(X_{T}\right) \mid X_{t}\right] \\
& =-c^{i}\left(A_{t}^{i}, X_{t}\right) \Delta+\mathbb{E}\left[U^{i}\left(X_{t}+\left(\sum_{j \in N} \mu_{j} A_{t}^{j}\right) \Delta+\epsilon_{t}\right) \mid X_{t}\right] .
\end{aligned}
$$

Note that by convolution the second term on the right-hand side is smooth in $A_{t}$. Therefore, the first- and second-order partial derivatives of $W_{t}^{i, \Delta}$ are

$$
\begin{aligned}
\frac{\partial}{\partial A_{t}^{i}} W_{t}^{i, \Delta} & =-\frac{\partial}{\partial A^{i}} c^{i} \Delta+\Delta \int U^{i}\left(X_{t}+\sum_{k} \mu_{k} A_{t}^{k} \Delta+\epsilon_{t}\right) \frac{\partial}{\partial \epsilon} \phi^{\Delta} d \epsilon_{t} \\
& =-\frac{\partial}{\partial A^{i}} c^{i} \Delta+\Delta \int U^{i}\left(X_{t}+\sum_{k} \mu_{k} A_{t}^{k} \Delta+\epsilon_{t}\right) \frac{\exp \left[\frac{-\epsilon_{t}^{2}}{2 \Delta}\right]}{\Delta \sqrt{2 \pi}} \frac{\epsilon_{t}}{\Delta} d \epsilon_{t} \\
& =-\frac{\partial}{\partial A^{i}} c^{i} \Delta+\mathbb{E}\left[\int U^{i}\left(X_{t}+\sum_{k} \mu_{k} A_{t}^{k} \Delta+\epsilon_{t}\right) \epsilon_{t} \mid X_{t}\right]
\end{aligned}
$$

and

$$
\begin{aligned}
\frac{\partial^{2}}{\partial A_{t}^{i} \partial A_{t}^{j}} W_{t}^{i, \Delta} & =-\frac{\partial^{2}}{\partial A^{i} \partial A^{j}} c^{i} \Delta+\Delta \int U^{i}\left(X_{t}+\sum_{k} \mu_{k} A_{t}^{k} \Delta+\epsilon_{t}\right) \epsilon_{t} \frac{\partial}{\partial \epsilon} \phi^{\Delta} d \epsilon_{t} \\
& =-\frac{\partial^{2}}{\partial A^{i} \partial A^{j}} c^{i} \Delta+\mathbb{E}\left[\int U^{i, \Delta}\left(X_{t}+\sum_{k} \mu_{k} A_{t}^{k} \Delta+\epsilon_{t}\right) \epsilon_{t} \epsilon_{t} \mid X_{t}\right],
\end{aligned}
$$

where $\phi^{\Delta}$ denotes the density of $\epsilon_{t}$.

We show that the equilibrium action profile at period $t$ is unique if $\Delta$ is small. To show this, we claim that the players' best response in period $t$ has a slope less than 1 if $\Delta$ is small enough; this ensures the uniqueness by the contraction mapping theorem. Note that the equilibrium action satisfies the first-order condition $\frac{\partial}{\partial A_{t}^{i}} W_{t}^{i, \Delta}=0$ in the case of an interior solution.

Because $U^{i}$ is Lipschitz continuous, there exists a constant $L>0$ such that

$$
\left|U^{i}(x+\epsilon)-U^{i}(x)\right| \leq L|\epsilon|
$$


for any $x, \epsilon$. The second-order partial derivatives are bounded in absolute value as follows:

$$
\begin{aligned}
\left|\frac{\partial^{2}}{\partial A_{t}^{i} \partial A_{t}^{j}} W_{t}^{i, \Delta}\right| & \leq \mathbb{E}\left[\left|\left(U^{i}\left(X_{t}+\sum_{k} \mu_{k} A_{t}^{k} \Delta+\epsilon_{t}\right)-U^{i}\left(X_{t}+\sum_{k} \mu_{k} A_{t}^{k} \Delta\right)\right) \epsilon_{t}^{2}\right|\right]+\left|\mathbb{E}\left[U^{i}\left(X_{t}+\sum_{k} \mu_{k} A_{t}^{k} \Delta\right) \epsilon_{t}^{2}\right]\right| \\
& \leq \mathbb{E}\left[\left(\left(U^{i}\left(X_{t}+\sum_{k} \mu_{k} A_{t}^{k} \Delta+\epsilon_{t}\right)-U^{i}\left(X_{t}+\sum_{k} \mu_{k} A_{t}^{k} \Delta\right)\right)^{2}\right]^{1 / 2} \mathbb{E}\left[\epsilon_{t}^{4}\right]^{1 / 2}\right. \\
& \leq \mathbb{E}\left[L^{2} 2 \epsilon_{t}^{2}\right]^{1 / 2}\left(\operatorname{Var}\left[\epsilon_{t}\right]^{2}\right)^{1 / 2} \\
& \leq L\left(2 \mathbb{E}\left[\epsilon_{t}^{4}\right]^{1 / 2}\right)^{1 / 2} \Delta \\
& =\sqrt{2} L \Delta^{3 / 2},
\end{aligned}
$$

which is based on $\mathbb{E}\left[\epsilon_{t}\right]=0$ and $\operatorname{Var}\left[\epsilon_{t}\right]=\Delta$; the Holder inequality was used on the second and fourth lines.

Let $K>0$ denote the lower bound of the second-order partial derivative of $c^{i}$ with respect to $A^{i}$. Then

$$
\begin{aligned}
\left|\frac{\partial^{2}}{\partial\left(A_{t}^{i}\right)^{2}} W_{t}^{i, \Delta}\right| & \geq|K \Delta-| \mathbb{E}\left[U^{i}\left(X_{t}+\sum_{k} \mu_{k} A_{t}^{k} \Delta+\epsilon_{t}\right) \epsilon_{t}^{2}\right]|| \\
& \geq\left|K \Delta-\sqrt{2} L \Delta^{3 / 2}\right|
\end{aligned}
$$

for sufficiently small $\Delta$. By the implicit function theorem applied to the first-order conditions, the slope of each player's best response in period $t$ is bounded as follows:

$$
\frac{\left|\frac{\partial^{2}}{\partial A_{t}^{i}, \partial A_{t}^{j}} W_{t}^{i, \Delta}\right|}{\left|\frac{\partial^{2}}{\partial\left(A_{t}^{i}\right)^{2}} W_{t}^{i, \Delta}\right|} \leq\left|\frac{\sqrt{2} L \Delta^{1 / 2}}{K-\sqrt{2} L \Delta^{1 / 2}}\right|
$$

which establishes the claim.

\section{B.5 Details for Example 3}

We show that no player has an incentive to deviate from the prescribed strategy. Fix any $t$ and current state $X_{t}$.

- Case 1: $X_{t} \geq(T-t) \theta$.

If there is no deviation, the terminal state is $X_{T}=X_{t}+(T-t) N$, which is strictly positive. After an arbitrary deviation on the part of player $i$ after time $t$, the state variable $X_{t^{\prime}}$ at any $t^{\prime}>t$ is strictly greater than $\left(T-t^{\prime}\right) \theta$ because of $\theta<$ $-N+1$. Thus, the terminal state after any deviation is still positive. Given this, 
the optimization problem reduces to

$$
\max _{A^{i}} \int_{t}^{T}\left(A_{s}^{i}-\kappa c\left(A_{s}^{i}\right)\right) d s
$$

and thus it is strictly optimal to choose $A_{t}^{i}=1$ at any time $t^{\prime} \geq t$.

- Case 2: $X_{t}<(T-t) \theta$.

If there is no deviation, the terminal state is $X_{T}=X_{t}-(T-t) N$, which is strictly negative. By the same argument as in Case 1, under an arbitrary deviation on the part of player $i$ after time $t$, the terminal state is strictly negative. Given this, the optimization problem reduces to

$$
\max _{A^{i}} \int_{t}^{T}\left(-c\left(A_{s}^{i}\right)\right) d s
$$

and thus it is strictly optimal to choose $A_{t}^{i}=0$ at any time $t^{\prime} \geq t$.

\section{B.6 Proof of Lemma 2}

Proof. By symmetry of the players, at the unique equilibrium $A=\left(A^{i}\right)_{i \in N}$, every player chooses the same action, namely $A_{t}^{i}=f\left(\beta_{t}(A)\right)$, where $f(\beta):=\arg \max _{\alpha \in \mathcal{A}}\left\{\sigma^{-1} \beta \alpha-\kappa c(\alpha)\right\}$ at almost all $t$ almost surely. Thus the equilibrium BSDE (6) reduces to a single dimension

$$
d W_{t}=-\left(N f\left(\beta_{t}\right) \sigma^{-1} \beta_{t}-\kappa c\left(f\left(\beta_{t}\right)\right)\right) d t+\sigma^{-1} \beta_{t} d X_{t}, \quad W_{T}=U\left(X_{T}\right) .
$$

Let $\hat{W}_{t}$ denote the continuation value process for this hypothetical single-agent problem

under the optimal policy $\hat{A}=\left(\hat{A}^{i}\right)_{i \in N}$. By construction, $\hat{A}_{t}^{i}=f\left(\hat{\beta}_{t}\right)$ for each $i \in N$ and almost all $t$ almost surely, where $(\hat{W}, \hat{\beta})$ follows the BSDE

$$
d \hat{W}_{t}=-\left(N f\left(\hat{\beta}_{t}\right) \sigma^{-1} \hat{\beta}_{t}-N \kappa \hat{c}\left(f\left(\hat{\beta}_{t}\right)\right)\right) d t+\sigma^{-1} \hat{\beta}_{t} d X_{t}, \quad \hat{W}_{T}=U\left(X_{T}\right) .
$$

By the assumption that $c(\cdot) \geq 0$, the comparison of the driver terms yields

$$
N f(\beta) \sigma^{-1} \beta-\kappa c(f(\beta)) \geq N f(\beta) \sigma^{-1} \beta-N \kappa c(f(b))
$$

for almost all $t$ almost surely. Because these BSDEs satisfy the quadratic growth condition, the comparison theorem (Lemma 4) implies $W_{0} \geq \hat{W}_{0}$.

To show the remaining inequality, consider the modified version of the hypothetical 
single-agent problem in which the agent maximizes $\mathbb{E}\left[U\left(X_{T}\right)-\int_{0}^{T} \kappa\left(\sum_{i} c\left(\hat{A}_{t}^{i}\right)-(N-\right.\right.$ 1) $\bar{c}) d t]$. Let $\tilde{W}_{t}$ denote the continuation value process under the optimal policy. Then

$$
d \tilde{W}_{t}=-\left(N f\left(\tilde{\beta}_{t}\right) \sigma^{-1} \tilde{\beta}_{t}-\kappa\left(N c\left(f\left(\tilde{\beta}_{t}\right)\right)+(N-1) \bar{c}\right)\right) d t+\sigma^{-1} \tilde{\beta}_{t} d X_{t}, \quad \tilde{W}_{T}=U\left(X_{T}\right),
$$

where $\tilde{\beta}_{t}$ is the corresponding sensitivity process. Comparing its driver to the driver term in (11), we obtain

$$
N f(\beta) \sigma^{-1} \beta-\kappa(N c(f(\beta))-(N-1) \bar{c}) \geq N f(\beta) \sigma^{-1} \beta-\kappa c(f(\beta))
$$

for almost all $t$ almost surely. By the comparison theorem (Lemma 4$), \tilde{W}_{0} \geq W_{0}$.

\section{B.7 Proof of Proposition 1}

Proof. We show that $\hat{W}_{0}$ is approximately greater than $\max _{x \in\left[X_{0}-N \underline{A} T, X_{0}+N \bar{A} T\right]} U(x)$ if both $\sigma$ and $\kappa$ are small. To see this, let $x^{*} \in \arg \max _{x \in\left[X_{0}-N \underline{A} T, X_{0}+N \bar{A} T\right]} U(x)$. Then, the expected payoff in the hypothetical single-agent problem under the constant policy $\left(\hat{A}_{t}^{i}=\frac{x^{*}-X_{0}}{N T}\right.$ for each $\left.i \in N\right)$ converges to $\max _{x \in\left[X_{0}-N \underline{A} T, X_{0}+N \bar{A} T\right]} U(x)$ as $\sigma, \kappa \rightarrow 0$. This expected payoff is lower than $\hat{W}_{0}$ by definition, and thus the Proposition follows by Lemma 1.

\section{B.8 Proof of Proposition 2}

Proof. First, we prove the following lemma, which restricts the sign of the $\beta_{t}$ process under the unique equilibrium for any values of the parameters, assuming that $U$ is increasing.

Lemma 9. Consider the team production game in which $U$ is increasing, and let $A=$ $\left(A^{i}\right)_{i \in N}$ be the unique Nash equilibrium. Then $\beta(A)_{t} \geq 0$ for almost all $t$ almost surely.

Proof. Note that the driver term in (11) satisfies the quadratic growth condition, and that the terminal payoff $U(x)$ is bounded, Lipschitz continuous, and increasing. Therefore, the claim follows by Corollary 3.5 and Remark 3.8 in dos Reis and dos Reis (2013).

For any $\beta \geq 0$, the term $\max _{\alpha} \Sigma^{-1} \beta \alpha-\kappa c(\alpha)$ is decreasing in $\kappa$ by the envelope theorem. Let $f(\beta ; \kappa):=\arg \max _{\alpha} \Sigma^{-1} \beta \alpha-\kappa c(\alpha)$. Thus, for each $\beta \geq 0$, the driver term in the equilibrium BSDE (11),

$$
(N-1) f(\beta ; \kappa) \Sigma^{-1} \beta+\max _{\alpha}\left\{\Sigma^{-1} \beta \alpha-\kappa c(\alpha)\right\}
$$


is decreasing in $\kappa$ and increasing in $N$. By Lemmas 4 and $9, W_{0}(A(\kappa, N)) \geq W_{0}(A(\tilde{\kappa}, N))$ for any $\kappa \leq \tilde{\kappa}$ and $N$, and $W_{0}(A(\kappa, N)) \leq W_{0}(A(\kappa, \tilde{N}))$ for any $N \leq \tilde{N}$ and $\kappa$.

\section{B.9 Proof of Proposition 3}

In the proof of Proposition 8, we construct an equilibrium strategy profile for general quadratic payoffs $U^{i}=\sum_{k=1,2}\left(\alpha_{i k}\left(x^{k}\right)^{2}+\beta_{i k} x^{k}\right)+\gamma_{i} x^{1} x^{2}$. This implies that the equilibrium actions and continuation values take the form $a^{i}\left(t, x^{1}, x^{2}\right)=\frac{1}{\bar{c}_{i}}\left(2 \alpha_{i i}(t) x^{i}+\beta_{i i}(t)+\right.$ $\left.\gamma_{i}(t) x^{j}\right)$ and $w^{i}\left(t, x^{1}, x^{2}\right)=\sum_{k=i, j}\left(\alpha_{i k}(t)\left(x^{k}\right)^{2}+\beta_{i k}(t) x^{k}\right)+\gamma_{i}(t) x^{i} x^{j}+\delta_{i}(t)$, where the coefficients are obtained by solving the system of ODEs (16) given in that proof. For the current game, we can directly verify the solution

$$
\begin{aligned}
& \alpha_{11}(t)=\alpha_{12}(t)=\gamma_{1}(t)=\alpha_{22}(t)=0 \\
& \beta_{11}(t)=\frac{T-t}{\bar{c}_{2}}, \quad \beta_{12}(t)=1, \quad \delta_{1}(t)=\frac{(T-t)^{3}}{3 \bar{c}_{1} \bar{c}_{2}^{2}} \\
& \alpha_{21}(t)=\frac{T-t}{2 \bar{c}_{2}}, \quad \beta_{22}(t)=\frac{(T-t)^{2}}{2 \bar{c}_{1} \bar{c}_{2}}, \quad \beta_{21}(t)=\frac{(T-t)^{3}}{2 \bar{c}_{1} \bar{c}_{2}^{2}} \\
& \gamma_{2}(t)=1, \quad \delta_{2}(t)=\frac{\sigma_{1}^{2}(T-t)^{2}}{4 \bar{c}_{2}}+\frac{3(T-t)^{5}}{20 \bar{c}_{1}^{2} \bar{c}_{2}^{3}}
\end{aligned}
$$

where the boundary conditions (17) given in the proof of Proposition 8 take the form $\beta_{12}(T)=\gamma_{2}(T)=1, \alpha_{11}(T)=\alpha_{12}(T)=\alpha_{21}(T)=\alpha_{22}(T)=\beta_{11}(T)=\beta_{21}(T)=\beta_{22}(T)=$ $\gamma_{1}(T)=\delta_{1}(T)=\delta_{2}(T)=0$.

Therefore, the equilibrium strategies are

$$
a^{1}\left(t, x^{1}, x^{2}\right)=\frac{T-t}{\bar{c}_{1} \bar{c}_{2}}, \quad a^{2}\left(t, x^{1}, x^{2}\right)=\frac{(T-t)^{2}}{2 \bar{c}_{1}\left(\bar{c}_{2}\right)^{2}}+\frac{x^{1}}{\bar{c}_{2}}
$$

For the continuation value functions,

$$
\begin{gathered}
w^{1}\left(t, x^{1}, x^{2}\right)=\frac{T-t}{\bar{c}_{2}} x^{1}+x^{2}+\frac{(T-t)^{3}}{3 \bar{c}_{1} \bar{c}_{2}^{2}} \\
w^{2}\left(t, x^{1}, x^{2}\right)=\frac{T-t}{2 \bar{c}_{2}}\left(x^{1}\right)^{2}+\frac{(T-t)^{3}}{2 \bar{c}_{1} \bar{c}_{2}^{2}} x^{1}+\frac{(T-t)^{2}}{2 \bar{c}_{1} \bar{c}_{2}} x^{2}+x^{1} x^{2}+\frac{\sigma_{1}^{2}(T-t)^{2}}{4 \bar{c}_{2}}+\frac{3(T-t)^{5}}{20 \bar{c}_{1}^{2} \bar{c}_{2}^{3}} .
\end{gathered}
$$




\section{Supplementary Appendix}

\section{Variations of the Model}

In this section, we consider several variations of the original model.

\section{C.1 Multiplicity with an Infinite Horizon}

We demonstrate that equilibrium may not be unique if there is no deterministic bounded deadline $T$. To make this point, we assume that the deadline $T$ is time of the first arrival of a Poisson process with fixed intensity $\lambda>0$. For simplicity, here we focus on the two-player case with one-dimensional state $d X_{t}=\sum_{i=1,2} A_{t}^{i} d t+\sigma d Z_{t}$.

Focusing on Markov strategies that depend on only the current state $X_{t}$, we can derive the following HJB equations:

$$
0=\max _{A^{i} \in \mathcal{A}^{i}}\left[-c^{i}\left(x, A^{i}\right)+\left(w^{i}\right)^{\prime}(x) \sum_{k=1,2} A^{k}+\frac{\sigma}{2}\left(w^{i}\right)^{\prime \prime}(x)+\lambda\left(U^{i}(x)-w^{i}(x)\right)\right],
$$

where $w^{i}(x)$ denote the continuation value of player $i$ when the state $X_{t}$ is equal to $x$. These equations can be written as

$$
\lambda w^{i}(x)=\max _{A^{i} \in \mathcal{A}^{i}}\left[\lambda U^{i}(x)-c^{i}\left(x, A^{i}\right)+\left(w^{i}\right)^{\prime}(x) \sum_{k=1,2} A^{k}+\frac{\sigma}{2}\left(w^{i}\right)^{\prime \prime}(x)\right] .
$$

Therefore, the model is equivalent to the infinite-horizon model in which each player receives flow payoff $\lambda U^{i}\left(X_{t}\right)-c^{i}\left(X_{t}, A^{i}\right)$ and has discount rate $\lambda$.

It is known in the literature that infinite-horizon differential games can have multiple equilibria in Markov strategies even if flow payoffs are independent of opponents' actions (e.g., Tsutsui and Mino (1990)). As a particularly simple example, consider the game in which the terminal payoff $U^{i}$ is constantly zero and the flow payoff $-c^{i}$ is given by $-\frac{1}{2}\left(\left(A_{t}^{i}\right)^{2}+4\left(A_{t}^{j}\right)^{2}+\gamma\left(X_{t}\right)^{2}\right)$ for some constant $\gamma>0$. Note that the uniqueness result in the main model still holds even if flow payoffs depend on opponents' actions in an additively separable manner (footnote 16). Based on Example 2 in Lockwood (1996), one can verify that there are two possible values of $\theta>0$ such that the strategy profile of the form $A_{t}^{i}=-\theta X_{t}$ is a (Markov perfect) equilibrium of this game when $\gamma$ is small. ${ }^{33}$

\footnotetext{
${ }^{33}$ Lockwood (1996) analyzes a deterministic model, in which $\sigma=0$. Under quadratic payoffs, any Markov
} 
This multiplicity contrasts with the uniqueness result under the original model with a finite horizon. Because there is no terminal-value condition on the continuation value $W_{t}^{i}$, the "backward induction" argument which drives the unique equilibrium in the original model does not work here.

\section{C.2 Discrete-Time Model}

In this subsection, we develop discrete-time models. Player $i$ chooses a control action $A_{t}^{i}$ at only the times $t=0, \Delta, 2 \Delta, \ldots, T-\Delta$, where $\Delta>0$ is the period length and $T / \Delta$ is assumed to be a natural number.

Player $i$ 's total payoff is given by

$$
U^{i}\left(X_{T}\right)-\Delta \sum_{t=0}^{T / \Delta} c^{i}\left(A_{t}^{i}\right)
$$

We retain Assumption 2 on $U^{i}$ and $c^{i}$. In the first subsection, we consider a discretized version of the original model. In the second subsection, we consider a Poisson noise model.

\section{C.2.1 Brownian Case}

We consider the same environment as in the main model, except that player $i$ 's action $A_{t}^{i}$ is assumed to remain constant in $[t, t+\Delta)$. To simplify the notation, we assume that $d=n$ and player $i$ chooses a one-dimensional action $A_{t}^{i}$ to influence the process $d X_{t}^{i}=A_{t}^{i} d t+d Z_{t}^{i}$. This assumption is not essential for any argument made here.

Note that the state evolves as

$$
X_{t+\Delta}^{i}=X_{t}^{i}+\Delta A_{t}^{i}+\epsilon_{t}^{i}
$$

for each $i \in N$ and $t=0, \Delta, \ldots, T-\Delta$, where $\epsilon_{t}^{i}$ is i.i.d noise that follows the normal distribution $N(0, \Delta)$.

We consider two cases: imperfect monitoring and perfect monitoring.

Let $\tilde{X}_{t}=\left(X_{0}, \ldots, X_{t}\right)$ denote a public history at period $t$, a sequence of state variables for all periods up to and including the one that begins at time $t$. We define player $i$ 's imperfect monitoring strategy $A^{i}$ as a function that, for period $t$, maps the public public history $\tilde{X}_{t-\Delta}$ to some element o $\mathcal{A}^{i}$. A Nash equilibrium under imperfect monitoring is

strategy equilibrium profile that is linear in the state constitutes an equilibrium of the stochastic model as long as the transversality condition is satisfied. 
a profile $\left(A^{i}\right)_{i \in N}$ of imperfect monitoring strategies such that each player maximizes her expected total payoff within the class of imperfect monitoring strategies. Note that the distribution of public histories at every period $t$ has full support. As usual, we do not distinguish between two strategies if for each period $t$, they specify the same action with probability 1 .

Let $\hat{X}_{t}=\left(\left(X_{0}, A_{0}^{i}\right)_{i \in N}, \ldots,\left(X_{t}, A_{t}^{i}\right)_{i \in N}\right)$ denote a full history at period $t$, that is, a sequence of the state variables and the actions chosen by the players for all periods up to and including the one that begins at time $t$. We define player $i$ 's perfect monitoring strategy $\hat{A}^{i}$ as a function that, for each period $t$, maps the full history $H_{t-\Delta}$ to some element of $\mathcal{A}^{i}$. A Nash equilibrium under perfect monitoring is a profile $\left(\hat{A}^{i}\right)_{i \in N}$ of perfect monitoring strategies such that each player maximizes her expected total payoff within the class of perfect monitoring strategies. We say that a profile $\left(\hat{A}^{i}\right)_{i \in N}$ of perfect monitoring strategies is outcome equivalent to a profile of imperfect monitoring strategies $\left(A^{i}\right)_{i \in N}$ if the distributions of the $X_{t}$ processes induced by the two strategy profiles coincide with probability 1 .

We first show that whenever there is a unique equilibrium under imperfect monitoring, then any equilibrium under perfect monitoring is essentially unique, that is, any equilibrium under perfect monitoring is outcome equivalent to the unique equilibrium under imperfect monitoring. This suggests that the monitoring imperfectness is not important for the equilibrium uniqueness result in the original model.

Proposition 5. Consider the discrete-period model with period length $\Delta$. Suppose that there is a unique Nash equilibrium $\left(A^{i}\right)_{i \in N}$ under imperfect monitoring. Then a Nash equilibrium under perfect monitoring exists. Moreover, any Nash equilibrium under perfect monitoring is outcome equivalent to $\left(A^{i}\right)_{i \in N}$.

Proof. Let $\left(A^{i}\right)_{i \in N}$ denote the unique Nash equilibrium under imperfect monitoring.

Step 1: $\left(A^{i}\right)_{i \in N}$ is a unique Nash equilibrium in Markov strategies.

Given a strategy profile $\left(A^{i}\right)_{i \in N}$, let $\tilde{W}^{i}\left(\tilde{X}_{t}\right)$ denote the expected continuation payoff for player $i$ at time $t$ conditional on public history $\tilde{X}_{t}$. Then

$$
\tilde{W}^{i}\left(\tilde{X}_{t}\right)=-c^{i}\left(A_{t}^{i}\left(\tilde{X}_{t}\right)\right) \Delta+\mathbb{E}\left[\tilde{W}^{i}\left(\left(\tilde{X}_{t}, X_{t}+A_{t}\left(\tilde{X}_{t}\right) \Delta+\epsilon_{t}\right)\right)\right]
$$

for each $t \in\{0, \Delta, \ldots, T-\Delta\}$ and $\tilde{X}_{t}$, with $\tilde{W}^{i}\left(\tilde{X}_{T}\right)=U^{i}\left(X_{T}\right)$.

Observe first that, at $t=T-\Delta$ and for almost all realizations of $\tilde{X}_{t},\left(A^{i}\left(\tilde{X}_{t}\right)\right)_{i \in N}$ 
should be a unique control action profile $A_{t}$ such that

$$
\left.\left.-c^{i}\left(A_{t}^{i}\right) \Delta+\mathbb{E}\left[U^{i}\left(X_{t}+A_{t} \Delta+\epsilon_{t}\right)\right)\right] \geq-c^{i}\left(\bar{A}_{t}^{i}\right) \Delta+\mathbb{E}\left[U^{i}\left(X_{t}+\left(\bar{A}_{t}^{i}, A_{t}^{-i}\right) \Delta+\epsilon_{t}\right)\right)\right] \forall \bar{A}_{t}^{i} \in \mathcal{A}^{i},
$$

for every $i$. (If not, we can construct multiple Nash equilibria under imperfect monitoring by specifying the different action profiles at histories of positive measure at $t=T-$ $\Delta$ and then solving backwards to determine the equilibrium actions at earlier periods.) Furthermore, since $U^{i}$ depend on only the terminal state, if we take any two histories $\tilde{X}_{t}, \tilde{X}_{t}^{\prime}$ such that the state variables at $t$ coincide, we must have $A^{i}\left(\tilde{X}_{t}\right)=A^{i}\left(\tilde{X}_{t}\right)$ for every $i$.

We iterate this argument backwards to earlier periods: Except for histories of measure 0 , the continuation value can be written as a function of time and state, $W_{t}\left(X_{t}\right)$, such that the equilibrium control action profile at period $t$ is a unique action profile $A_{t}$ with the property that

$\left.\left.-c^{i}\left(A_{t}^{i}\right) \Delta+\mathbb{E}\left[W_{t+\Delta}^{i}\left(X_{t}+A_{t} \Delta+\epsilon_{t}\right)\right)\right] \geq-c^{i}\left(\bar{A}_{t}^{i}, X_{t}\right) \Delta+\mathbb{E}\left[W_{t+\Delta}^{i}\left(X_{t}+\left(\bar{A}_{t}^{i}, A_{t}^{-i}\right) \Delta+\epsilon_{t}\right)\right)\right] \forall \bar{A}_{t}^{i} \in \mathcal{A}^{i}$

for every $i$.

\section{Step 2: Outcome equivalence}

Next, we take any Nash equilibrium $\left(\hat{A}^{i}\right)_{i \in N}$ under perfect monitoring. Let $\hat{W}^{i}\left(\hat{X}_{t}\right)$ denote the expected continuation payoff for player $i$ at time $t$ conditional on history $\hat{X}_{t}$. Note that at time $t=T-\Delta$,

$\left.\left.-c^{i}\left(A^{i}\left(\hat{X}_{t}\right)\right) \Delta+\mathbb{E}\left[U^{i}\left(X_{t}+A_{t}\left(\hat{X}_{t}\right) \Delta+\epsilon_{t}\right)\right)\right] \geq-c^{i}\left(\bar{A}_{t}^{i}\right) \Delta+\mathbb{E}\left[U^{i}\left(X_{t}+\left(\bar{A}_{t}^{i}, A_{t}^{-i}\left(\hat{X}_{t}\right)\right) \Delta+\epsilon_{t}\right)\right)\right] \forall \bar{A}_{t}^{i} \in \mathcal{A}^{i}$

holds with probability 1 for every $i \in N$. Thus, by the argument in Step $1, \hat{A}^{i}\left(\hat{X}_{t}\right)=$ $A^{i}\left(\tilde{X}_{t}\right)$ holds with probability 1 for each $i$ (Here we are comparing two strategy profiles under the common probability space in which the distribution of $\left(\epsilon_{t}\right)_{t=0, \ldots, T}$ is fixed.) This implies $\tilde{W}^{i}\left(\tilde{X}_{t}\right)=\hat{W}^{i}\left(\hat{X}_{t}\right)$ with probability 1 .

Based on the argument in Step 1, we iterate this argument backwards to earlier periods: Except for histories of measure 0, the continuation value can be written as a function of time and state, $\hat{W}_{t}\left(X_{t}\right)$, such that the equilibrium control action profile at period $t$ is a 
unique action profile $A_{t}$ with the property that

$$
\left.\left.-c^{i}\left(A_{t}^{i}\right) \Delta+\mathbb{E}\left[\hat{W}_{t+\Delta}^{i}\left(X_{t}+A_{t} \Delta+\epsilon_{t}\right)\right)\right] \geq-c^{i}\left(\bar{A}_{t}^{i}, X_{t}\right) \Delta+\mathbb{E}\left[\hat{W}_{t+\Delta}^{i}\left(X_{t}+\left(\bar{A}_{t}^{i}, A_{t}^{-i}\right) \Delta+\epsilon_{t}\right)\right)\right] \forall \bar{A}_{t}^{i} \in \mathcal{A}^{i}
$$

for every $i$. This leads to $\hat{A}^{i}\left(\hat{X}_{t}\right)=A^{i}\left(\tilde{X}_{t}\right)$ and $\tilde{W}^{i}\left(\tilde{X}_{t}\right)=\hat{W}^{i}\left(\hat{X}_{t}\right)$ with probability 1 for each period $t$ and $i \in N$. This completes the proof.

The next result provides a uniqueness result for small $\Delta$ under a restriction on the equilibrium continuation value functions:

Proposition 6. Consider the discrete-time game described above. Assume that there exist constants $M>0$ and $\delta \in\left(0, \frac{1}{2}\right)$ such that for each $\Delta$, any equilibrium continuation value $W^{i, \Delta}\left(\tilde{X}_{t}\right)$ is Lipschitz continuous with coefficient less than $M \Delta^{\delta-\frac{1}{2}}$. Then for small enough $\Delta$, there is a unique equilibrium.

The proof is based on repeating the argument in Appendix B.4 and thus is omitted. The result is not entirely satisfactory, in that it restricts the asymptotic behavior of the slopes of the continuation value functions. While we conjecture that this assumption is unnecessary, this conjecture has not yet been proved. In this paper, we have circumvented this issue by formulating the game directly in continuous time.

\section{C.2.2 Poisson Case}

Let $\lambda^{i}: \mathcal{A}^{i} \rightarrow \mathbb{R}_{++}$be a bounded, differentiable function with a bounded derivative, and suppose that the state variable $X_{t}^{i}$ follows a compound Poisson process

$$
X_{t}^{i}=\sum_{l=1}^{N_{t}^{i}} D_{l}^{i}
$$

where $N_{t}^{i}$ is an inhomogeneous Poisson process with intensity $\lambda^{i}\left(A_{t}^{i}\right)>0$ at time $t$, and the jump sizes $\left(D_{l}^{i}\right)_{l=1,2, \ldots, N_{t}^{i}}$ are i.i.d and follow cumulative distribution $G^{i}$. We approximate this environment with the following discretized model: Choose $\Delta$ sufficiently small so that $\max _{i, A^{i}} \lambda^{i}\left(A^{i}\right) \Delta \leq 1$. At each period $t, X_{t+\Delta}^{i}=X_{t}^{i}$ holds with probability $1-\Delta \lambda^{i}\left(A_{t}^{i}\right)$. Conditional on $X_{t+\Delta}^{i} \neq X_{t}^{i}$, the jump size $D_{t}^{i}:=X_{t+\Delta}^{i}-X_{t}^{i}$ follows the distribution $G^{i}$. We define a strategy (in imperfect monitoring) as a function that, for $t=0, \Delta, 2 \Delta, \ldots, T-\Delta$, maps public history $\left(X_{0}, X_{\Delta}, \ldots, X_{t}\right)$ to some element of $\mathcal{A}^{i}$ as in the previous subsection. The notion of Nash equilibrium is defined likewise. 
Proposition 7. Consider the discrete-time model with Poisson noise. There is a unique Nash equilibrium if $\Delta$ is small enough.

We note that the analogue of Proposition 6 holds, so that the uniqueness result is intact even if we consider the perfect monitoring case.

\section{Quadratic Games}

In this subsection, we analyze a specific class of games in which the terminal payoffs and adjustment costs are quadratic functions, and we show that equilibrium PDEs can be simplified to ODEs. Because of the tractability of quadratic games, they have been widely employed in applications. A drawback to this approach is that it involves unbounded terminal payoffs and unbounded control ranges, which violate the assumptions for our main theorems; the uniqueness of equilibrium is an open question. In this section, we assume the two-player case as in Supplementary Appendix C.1 for simplicity.

We consider a quadratic game that has the following terminal payoff function and cost function:

$$
U^{i}(X)=\sum_{k=1,2}\left(\bar{\alpha}_{i k}\left(X^{k}\right)^{2}+\bar{\beta}_{i k} X^{k}\right)+\bar{\gamma}_{i} X^{1} X^{2}, \quad c^{i}(a)=\frac{\bar{c}_{i}}{2} a^{2}
$$

where $\bar{\alpha}_{i k}, \bar{\beta}_{i k}, \bar{\gamma}_{i}, \bar{c}_{i}$ are fixed constants such that $\bar{c}_{i}>0$. We also allow for unbounded controls: $\mathcal{A}^{i}=\mathbb{R}$. Proposition 8 states that an equilibrium is linear in the state variables, where the coefficients follow ODEs.

Proposition 8. Consider a quadratic game. Let $\left(\alpha_{i k}(t), \beta_{i k}(t), \gamma_{i}(t), \delta_{i}(t)\right)_{i, k=1,2, t \in[0, T]}$ be a solution to the system of ordinal differential equations

$$
\begin{aligned}
& \dot{\alpha}_{i i}(t)=-\frac{2\left(\alpha_{i i}(t)\right)^{2}}{\bar{c}_{i}}-\frac{\gamma_{i}(t) \gamma_{j}(t)}{\bar{c}_{j}}, \quad \dot{\alpha}_{i j}(t)=-\frac{\gamma_{i}^{2}(t)}{2 \bar{c}_{i}}-\frac{4 \alpha_{i j}(t) \alpha_{j j}(t)}{\bar{c}_{j}} \\
& \dot{\beta}_{i i}(t)=-\frac{2 \alpha_{i i}(t) \beta_{i i}(t)}{\bar{c}_{i}}-\frac{\beta_{j j}(t) \gamma_{i}(t)+\beta_{i j}(t) \gamma_{j}(t)}{\bar{c}_{j}}, \dot{\beta}_{i j}(t)=-\frac{\beta_{i i}(t)}{\bar{c}_{i}} \gamma_{i}(t)-\frac{2\left(\alpha_{i j}(t) \beta_{j j}(t)+\alpha_{j j}(t) \beta_{i j}(t)\right)}{\bar{c}_{j}} \\
& \dot{\gamma}_{i}(t)=-\frac{2 \alpha_{i i}(t) \gamma_{i}(t)}{\bar{c}_{i}}-\frac{2\left(\alpha_{i j}(t) \gamma_{j}(t)+\alpha_{j j}(t) \gamma_{i}(t)\right)}{\bar{c}_{j}}, \dot{\delta}_{i}(t)=-\left(\sigma_{1}\right)^{2} \alpha_{i 1}-\left(\sigma_{2}\right)^{2} \alpha_{i 2}-\frac{\beta_{i i}^{2}}{2 \bar{c}_{i}}-\frac{\beta_{i j} \beta_{j j}}{\bar{c}_{j}}
\end{aligned}
$$

associated with the boundary conditions

$$
\alpha_{i k}(T)=\bar{\alpha}_{i k}, \quad \bar{\beta}_{i k}(T)=\beta_{i k}, \quad \bar{\gamma}_{i}(T)=\gamma_{i}, \quad \delta_{i}(T)=0
$$


Then a Nash equilibrium strategy takes the form

$$
a^{i}\left(t, x^{1}, x^{2}\right)=\frac{1}{\bar{c}_{i}}\left(2 \alpha_{i i}(t) x^{i}+\beta_{i i}(t)+\gamma_{i}(t) x^{j}\right)
$$

\section{E Proofs of Results in the Appendix}

\section{E.1 Uniformly Parabolic PDEs}

The lemma below on uniformly parabolic PDEs is taken directly from Theorem 7.1 on p.596 in Ladyzenskaja, Solonnikov, and Ural'ceva (1995). It gives a set of sufficient conditions that ensures a unique solution to a system of second-order PDEs. In the statement below, $\mathbf{u}=\left(u^{i}\right)_{i=1}^{n}: \mathbb{R}^{d} \times[0, T] \rightarrow \mathbb{R}^{n}$ is the function to be determined, and the subscripts represent partial derivatives, that is, $\mathbf{u}_{\mathbf{t}}=\left(\frac{\partial u^{i}}{\partial t}\right)_{i=1}^{n}, \mathbf{u}_{k l}=\left(\frac{\partial^{2} u^{i}}{\partial x^{k} \partial x^{l}}\right)_{i=1}^{n}$, $\mathbf{u}_{k}=\left(\frac{\partial u^{i}}{\partial x^{k}}\right)_{i=1}^{n}$ and $\mathbf{u}_{\mathbf{x}}=\left(\mathbf{u}_{k}\right)_{k=1}^{d}$. Let $\mathbb{H}$ denote the set of $n$-dimensional functions $\mathbf{u}(x, t)$ that are continuous, together with all derivatives of the form $D_{t}^{r} D_{x}^{s}$ with $2 r+s<3$.

Lemma 10. Consider the following $n$-dimensional PDE system in $\mathbf{u}=\left(u^{i}\right)_{i=1}^{n}: \mathbb{R}^{d} \times$ $[0, T] \rightarrow \mathbb{R}^{n}:$

$$
\mathbf{u}_{t}-\sum_{k, l} \alpha^{k l}(x, t, \mathbf{u}) \mathbf{u}_{k l}+\sum_{k} \delta^{k}\left(x, t, \mathbf{u}, \mathbf{u}_{\mathbf{x}}\right) \mathbf{u}_{k}+\gamma\left(x, t, \mathbf{u}, \mathbf{u}_{\mathbf{x}}\right)=0, \quad \mathbf{u}(x, T)=U(x),
$$

where $\alpha^{k l}: \mathbb{R}^{d} \times[0, T] \times \mathbb{R}^{n} \rightarrow \mathbb{R}, \delta^{k}: \mathbb{R}^{d} \times[0, T] \times \mathbb{R}^{n} \times \mathbb{R}^{n \times d} \rightarrow \mathbb{R}, \gamma: \mathbb{R}^{d} \times[0, T] \times \mathbb{R}^{n} \times$ $\mathbb{R}^{n \times d} \rightarrow \mathbb{R}^{n}$, and $U: \mathbb{R}^{d} \rightarrow \mathbb{R}^{n}$ are exogenous functions, $k, l=1, \ldots, d$.

Then there exists a unique solution to the above system in $\mathbb{H}$ if the following conditions are satisfied:

1. There exist $M_{1}, M_{2} \geq 0$ such that

$$
\gamma^{i}(x, t, \mathbf{u}, \mathbf{0}) u^{i} \geq-M_{1}|\mathbf{u}|^{2}-M_{2}, i, j=1, \ldots, n
$$

2. There exist constants $M_{3}, \mu, \nu>0$ such that

(a) (Uniform Parabolicity)

$$
\nu \leq \frac{1}{|\xi|^{2}} \sum_{k, l} \alpha^{k l}(t, x, \mathbf{u}) \xi^{k} \xi^{l} \leq \mu, k, l=1, \ldots, d
$$


(b)

$$
\left|\delta^{k}(x, t, \mathbf{u}, \mathbf{p})\right| \leq \mu(1+|\mathbf{p}|)^{2}, k=1, \ldots, d
$$

(c)

$$
|\gamma(x, t, \mathbf{u}, \mathbf{p})| \leq \frac{M_{3}}{|\mathbf{p}|}(1+|\mathbf{p}|)^{2}
$$

$$
\left|\frac{\partial \alpha^{k l}}{\partial x^{k}}(x, t, \mathbf{u}, \mathbf{p}), \frac{\partial \alpha^{k l}}{\partial u^{i}}(x, t, \mathbf{u}, \mathbf{p})\right| \leq \mu, k, l=1, \ldots, d
$$

3. The first-order partial derivatives of $\alpha^{k l}, \delta^{k}, \gamma$ with respect to $(t, x, \mathbf{u}, \mathbf{p})$ are continuous, as are the second-order partial derivatives $\frac{\partial^{2} \alpha^{k l}}{\partial u^{i} \partial u^{j}}, \frac{\partial^{2} \alpha^{k l}}{\partial u^{i} \partial x^{k}}, \frac{\partial^{2} \alpha^{k l}}{\partial u^{l} \partial t}, \frac{\partial^{2} \alpha^{k l}}{\partial x^{k} \partial u^{n}}$.

4. $U(\cdot)$ is $C^{3}$ and has bounded derivatives.

\section{E.2 Proof of Proposition 4}

The proof consists of two steps.

Step 1: Construction of a bounded sensitivity equilibrium

Lemma 11. The PDE system (8) with the terminal condition has a unique solution $\tilde{w}$ that is $C^{3, b}$.

Proof. Let us reproduce the PDE system:

$0=\frac{\partial w^{i}(t, x)}{\partial t}+\frac{1}{2} \sum_{l, m=1}^{d} \sum_{k=1}^{d} \sigma_{l k} \sigma_{m k} \frac{\partial^{2} w^{i}(t, x)}{\partial x^{l} \partial x^{m}}+\left(\sum_{j \in N} \mu_{j} a^{j}+\mu_{0}(x)\right) \cdot \nabla_{x} w^{i}(t, x)-c^{i}\left(a^{i}, x\right)$ where $a^{i}(t, x)=f^{i}\left(\mu_{i}(x) \cdot \nabla_{x} w^{i}(t, x), x\right)$ for all $(t, x)$, with a terminal condition $w^{i}(T, x)=$ $U^{i}(x)$. To see how the notations in Lemma 10 correspond to the current case, define the following quantities for $i=1, \ldots, n$ and $k, l, m=1, \ldots, d$ :

$$
\alpha^{l m}=\frac{1}{2} \sum_{k=1}^{d} \sigma_{l k} \sigma_{m k}, \delta^{k}=\left(\sum_{j \in N} \mu_{j}^{k} f^{j}\left(\nabla_{x} w^{j}(t, x), x\right)+\mu_{0}^{k}(x)\right), \gamma^{i}=-c^{i}\left(f^{i}\left(\nabla_{x} w, x\right), x\right) .
$$

Note that $f^{i}$ is continuously differentiable and bounded, by Assumption 3 and the implicit function theorem. We now verify that each of the conditions of Lemma 10 is satisfied.

Condition (1) holds because $c^{i}\left(f^{i}, x\right)$ is bounded. Condition (2a) follows from the fact that $\Sigma$ is invertible (see footnote 31 ). Condition $(2 \mathrm{~d})$ is clearly satisfied, because the 
$\alpha^{l m}$ are constants. If we take $\mu$ to be sufficiently large, then condition (2b) is satisfied because $f^{i}$ is bounded. Likewise, with a sufficiently large $M_{3},(2 \mathrm{c})$ is satisfied because $c^{i}$ is bounded. The continuity assumptions in (3) follow from the continuity of the firstorder partial derivatives of $c^{i}$ and $f^{i}$. Finally, (4) is satisfied because $U^{i}(\cdot)$ is $C^{3}$ and has bounded derivatives.

Based on the uniqueness of the solution, we construct a particular strategy profile $\tilde{A}$ defined as

$$
\tilde{A}_{t}^{i}=f^{i}\left(\nabla_{x} \tilde{w}\left(t, X_{t}\right), X_{t}\right) .
$$

Lemma 12. $\tilde{A}$ is an equilibrium with bounded sensitivity strategies.

Proof. Treating $\nabla_{x} w^{i}$ as a column-vector-valued function, for each $i$ define

$$
\beta_{t}^{i}(\tilde{A}):=\Sigma^{\prime} \nabla_{x} w^{i}\left(t, X_{t}\right)
$$

(equivalently $\nabla_{x} w^{i}\left(t, X_{t}\right)=\Sigma^{-1^{\prime}} \beta_{t}^{i}(\tilde{A})$ ).

By applying Ito's formula, we can confirm that $(W, \beta(\tilde{A}))$ indeed solves the equilibrium BSDE

$d W_{t}^{i}=\left(c^{i}\left(f^{i}\left(\nabla_{x} w^{i}\left(t, X^{t}\right), X_{t}\right), X_{t}\right)-\beta_{t}^{i}(\tilde{A}) \cdot \Sigma^{-1} \sum_{j} \mu_{j} f^{j}\left(\nabla_{x} w^{j}\left(t, X_{t}\right), X_{t}\right)+\mu_{0}\left(X_{t}\right)\right) d t+\beta_{t}^{i}(\tilde{A}) \cdot \Sigma^{-1} d X_{t}$ with $W_{T}^{i}=U^{i}\left(X_{T}\right)$.

Note that the first-order partial derivatives of $w^{i}$ with respect to $x$ are uniformly bounded. The results above verify that $\tilde{A}$ is a regular equilibrium strategy.

Step 2: Uniqueness of a bounded sensitivity equilibrium

Suppose there exist two bounded sensitivity equilibrium strategy profiles, $A$ and $\tilde{A}$. Let $(W, \beta)$ and $(\tilde{W}, \tilde{\beta})$ denote the corresponding equilibrium value and sensitivity processes. Note that the driver terms of the BSDE take the form

$$
g^{i}(t, w, b)=c^{i}\left(f^{i}(b, x), x\right)-b^{i} \cdot \Sigma^{-1}\left(\sum_{i} \mu_{i} f^{i}(b, x)+\mu_{0}(x)\right),
$$


which is continuously differentiable in $b$. Define a function $h: \mathbb{R}^{n \times n} \rightarrow \mathbb{R}^{n \times n}$ by

$$
h^{i, j}(b)=\left\{\begin{array}{lll}
M & \text { if } & b^{i, j} \geq M \\
b^{i, j} & \text { if } & b^{i, j} \in(-M, M) \\
-M & \text { if } & b^{i, j} \leq-M .
\end{array}\right.
$$

for each $i, j$. By the boundedness of $\beta$ and $\tilde{\beta}$, if we take a sufficiently large $M>0,(W, \beta)$ and $(\tilde{W}, \tilde{\beta})$ are solutions to the modified BSDE with driver terms

$$
\bar{g}^{i}(t, w, b)=c^{i}\left(f^{i}(h(b), x)\right)-h^{i}(b) \cdot \Sigma^{-1}\left(\sum_{i} \mu_{i} f^{i}(h(b), x)+\mu_{0}(x)\right)
$$

and terminal condition $U\left(X_{T}\right)$. This driver satisfies the uniform Lipschitz condition in Lemma 3 (see Appendix B.1.1) and thus there is a unique solution to the modified BSDE. Thus $(W, \beta)=(\tilde{W}, \tilde{\beta})$, which implies $A=\tilde{A}$.

\section{E.3 Proof of Proposition 7}

Proof. To simplify the notation, we consider only the two-player case. By the boundedness of $c^{i}$ and $U^{i}$, there is $M>0$ such that any equilibrium continuation payoff is bounded in absolute value by $M$ uniformly in $\Delta, A, t$, and the history. Let $M^{\prime}:=\inf _{i, A^{i}, X} \frac{\partial^{2}\left(c^{i}\right)\left(A^{i}, X\right)}{\partial\left(A^{i}\right)^{2}}$, which is strictly positive. Also, let $L:=\sup _{i, A^{i}}\left|\left(\lambda^{i}\right)^{\prime}\left(A^{i}\right)\right|$.

Fix any $\Delta$ and any Nash equilibrium strategy profile $A$. Take any $t$ and history $h_{t}=\left(X_{0}, \ldots, X_{t}\right)$. Let $\left(A_{t}^{i}, A_{t}^{j}\right)$ denote the action profile specified in the strategy profile. Then $A_{t}^{i}$ is a best-response against $A_{t}^{j}$ that maximizes

$$
\begin{aligned}
& -c^{i}\left(a^{i}, X_{t}\right) \Delta+\mathbb{E}\left[W^{i}\left(\left(h_{t}, X_{t+\Delta}\right)\right) \mid A_{t}^{j}, a^{i}\right] \\
= & -c^{i}\left(a^{i}, X_{t}\right) \Delta+\left(1-\Delta \lambda^{i}\left(a^{i}\right)\right) \mathbb{E}\left[W^{i}\left(\left(h_{t},\left(X_{t}^{i}, X_{t+\Delta}^{j}\right)\right)\right) \mid A_{t}^{j}\right] \\
& +\Delta \lambda^{i}\left(a^{i}\right) \int \mathbb{E}\left[W^{i}\left(\left(h_{t},\left(X_{t}^{i}+D_{t}^{i}, X_{t+\Delta}^{j}\right)\right)\right) \mid A_{t}^{j}\right] d G^{i}\left(D_{t}^{i}\right)
\end{aligned}
$$

over $a^{i} \in \mathcal{A}^{i}$, where $W^{i}\left(h_{t^{\prime}}\right)$ denotes the equilibrium continuation payoff at history $h_{t^{\prime}}$. 
The first derivative in $A_{t}^{i}$ takes the form

$$
\begin{aligned}
& -\frac{\partial c^{i}\left(A_{t}^{i}, X_{t}\right)}{\partial A^{i}} \Delta+\Delta\left(\lambda^{i}\right)^{\prime}\left(A_{t}^{i}\right) \mathbb{E}\left[\int W^{i}\left(\left(h_{t},\left(X_{t}^{i}+D_{t}^{i}, X_{t+\Delta}^{j}\right)\right)\right) d G^{i}\left(D_{t}^{i}\right)-W^{i}\left(\left(h_{t},\left(X_{t}^{i}, X_{t+\Delta}^{j}\right)\right)\right) \mid A_{t}^{j}\right] \\
& =-\frac{\partial c^{i}\left(A_{t}^{i}, X_{t}\right)}{\partial A^{i}} \Delta+\Delta\left(\lambda^{i}\right)^{\prime}\left(A_{t}^{i}\right) \Delta \lambda^{j}\left(A_{t}^{j}\right)\left(\int W^{i}(+,+) d G^{i} d G^{j}-\int W^{i}(0,+) d G^{j}\right) \\
& \quad+\Delta\left(\lambda^{i}\right)^{\prime}\left(A_{t}^{i}\right)\left(1-\Delta \lambda^{j}\left(A_{t}^{j}\right)\right)\left(\int W^{i}(+, 0) d G^{i}-W^{i}(0,0)\right),
\end{aligned}
$$

where we used the shorthand notation of the continuation value where + or 0 indicates whether each state variable admits a jump within the period or not.

For $j \neq i$, the derivative of left hand side with respect to $A_{t}^{j}$ is

$$
\begin{aligned}
& \Delta\left(\lambda^{i}\right)^{\prime}\left(A_{t}^{i}\right) \Delta\left(\lambda^{j}\right)^{\prime}\left(A_{t}^{j}\right)\left(\int W^{i}(+,+) d G^{i} d G^{j}-\int W^{i}(0,+) d G^{j}\right) \\
& \left.-\Delta\left(\lambda^{i}\right)^{\prime}\left(A_{t}^{i}\right) \Delta\left(\lambda^{j}\right)^{\prime}\left(A_{t}^{j}\right)\right)\left(\int W^{i}(+, 0) d G^{i}-W^{i}(0,0)\right),
\end{aligned}
$$

which is bounded by $4 \Delta^{2} L^{2} M$ in the absolute value.

As in Appendix B.4, using the implicit function theorem, the best-response of $i$ 's action conditional on this history against the opponent's choice $A_{t}^{j}$ has a slope less than 1 if $\Delta$ is sufficiently small. Because this bound on $\Delta$ is uniform in all histories, we can apply the contraction mapping argument from the last period to show that a Nash equilibrium is unique.

\section{E.4 Proof of Proposition 8}

Proof. We first solve $\left(\alpha_{i k}(t), \beta_{i k}(t), \gamma_{i}(t), \delta_{i}(t)\right)_{i, k=1,2, t \in[0, T]}$ by solving the system of ordinal differential equations

$$
\begin{aligned}
& \dot{\alpha}_{i i}(t)=-\frac{2\left(\alpha_{i i}(t)\right)^{2}}{\bar{c}_{i}}-\frac{\gamma_{i}(t) \gamma_{j}(t)}{\bar{c}_{j}}, \quad \dot{\alpha}_{i j}(t)=-\frac{\gamma_{i}^{2}(t)}{2 \bar{c}_{i}}-\frac{4 \alpha_{i j}(t) \alpha_{j j}(t)}{\bar{c}_{j}} \\
& \dot{\beta}_{i i}(t)=-\frac{2 \alpha_{i i}(t) \beta_{i i}(t)}{\bar{c}_{i}}-\frac{\beta_{j j}(t) \gamma_{i}(t)+\beta_{i j}(t) \gamma_{j}(t)}{\bar{c}_{j}}, \dot{\beta}_{i j}(t)=-\frac{\beta_{i i}(t)}{\bar{c}_{i}} \gamma_{i}(t)-\frac{2\left(\alpha_{i j}(t) \beta_{j j}(t)+\alpha_{j j}(t) \beta_{i j}(t)\right)}{\bar{c}_{j}} \\
& \dot{\gamma}_{i}(t)=-\frac{2 \alpha_{i i}(t) \gamma_{i}(t)}{\bar{c}_{i}}-\frac{2\left(\alpha_{i j}(t) \gamma_{j}(t)+\alpha_{j j}(t) \gamma_{i}(t)\right)}{\bar{c}_{j}}, \dot{\delta}_{i}(t)=-\left(\sigma_{1}\right)^{2} \alpha_{i 1}-\left(\sigma_{2}\right)^{2} \alpha_{i 2}-\frac{\beta_{i i}^{2}}{2 \bar{c}_{i}}-\frac{\beta_{i j} \beta_{j j}}{\bar{c}_{j}}
\end{aligned}
$$


associated with the boundary conditions:

$$
\alpha_{i k}(T)=\bar{\alpha}_{i k}, \quad \bar{\beta}_{i k}(T)=\beta_{i k}, \quad \bar{\gamma}_{i}(T)=\gamma_{i}, \quad \delta_{i}(T)=0 .
$$

Guess the functional form of value function by

$$
w^{i}\left(t, x^{1}, x^{2}\right)=\sum_{k=i, j}\left(\alpha_{i k}(t)\left(x^{k}\right)^{2}+\beta_{i k}(t) x^{k}\right)+\gamma_{i}(t) x^{i} x^{j}+\delta_{i}(t)
$$

Substituting this into the HJB equations

$$
\begin{aligned}
0= & \sum_{k=i, j}\left(\dot{\alpha}_{i k}\left(x^{k}\right)^{2}+\dot{\beta}_{i k} x^{k}\right)+\dot{\gamma}_{i} x^{i} x^{j}+\dot{\delta}_{i}+\left(\sigma_{1}\right)^{2} \alpha_{i 1}+\left(\sigma_{2}\right)^{2} \alpha_{i 2}+\frac{1}{2 \bar{c}_{i}}\left(2 \alpha_{i i} x^{i}+\beta_{i i}+\gamma_{i} x^{j}\right)^{2} \\
& +\frac{1}{\bar{c}_{j}}\left(2 \alpha_{i j} x^{j}+\beta_{i j}+\gamma_{i} x^{i}\right)\left(2 \alpha_{j j} x^{j}+\beta_{j j}+\gamma_{j} x^{i}\right) \\
= & \left(x^{i}\right)^{2}\left(\dot{\alpha}_{i i}+\frac{2 \alpha_{i i}^{2}}{\bar{c}_{i}}+\frac{\gamma_{i} \gamma_{j}}{\bar{c}_{j}}\right)+\left(x^{j}\right)^{2}\left(\dot{\alpha}_{i j}+\frac{\gamma_{i}^{2}}{2 \bar{c}_{j}}+\frac{4 \alpha_{i j} \alpha_{j j}}{\bar{c}_{j}}\right)+x^{i} x^{j}\left(\dot{\gamma}_{i}+\frac{2 \alpha_{i i} \gamma_{i}}{\bar{c}_{i}}+\frac{2}{\bar{c}_{j}}\left(\alpha_{i j} \gamma_{j}+\alpha_{j j} \gamma_{i}\right)\right) \\
& +x^{i}\left(\dot{\beta}_{i i}+\frac{2 \alpha_{i i} \beta_{i i}}{\bar{c}_{i}}+\frac{\beta_{j j} \gamma_{i}+\beta_{i j} \gamma_{j}}{\bar{c}_{j}}\right)+x^{j}\left(\dot{\beta}_{i j}+\frac{\beta_{i i} \gamma_{i}}{\bar{c}_{i}}+\frac{2\left(\alpha_{i j} \beta_{j j}+\alpha_{j j} \beta_{i j}\right)}{\bar{c}_{j}}\right) \\
& +\left(\dot{\delta}_{i}+\left(\sigma_{1}\right)^{2} \alpha_{i 1}+\left(\sigma_{2}\right)^{2} \alpha_{i 2}+\frac{\beta_{i i}^{2}}{2 \bar{c}_{i}}+\frac{\beta_{i j} \beta_{j j}}{\bar{c}_{j}}\right)
\end{aligned}
$$

where we have omitted the argument $t$ for coefficient functions. By the proposed ODE, the right hand side becomes zero for every $x^{1}$ and $x^{2}$. Also, the boundary condition holds with

$$
w^{i}\left(T, x^{1}, x^{2}\right)=\sum_{k=i, j}\left(\bar{\alpha}_{i k}\left(x^{k}\right)^{2}+\bar{\beta}_{i k} x^{k}\right)+\bar{\gamma}_{i} x^{i} x^{j}
$$

which verifies the solution.

Finally, by Theorem 1, the equilibrium action is given by

$$
a^{i}\left(t, x^{1}, x^{2}\right)=\frac{w_{i}^{i}\left(t, x^{1}, x^{2}\right)}{\bar{c}_{i}}=\frac{1}{\bar{c}_{i}}\left(2 \alpha_{i i}(t) x^{i}+\beta_{i i}(t)+\gamma_{i}(t) x^{j}\right)
$$

\section{References}

Admati, A., And M. Perry (1991): "Joint Projects without Commitment," Review of Economic Studies, 58(2), 259-276. 
Battaglini, M., S. Nunnari, and T. R. Palfrey (2014): "Dynamic Free Riding with Irreversible Investments," American Economic Review, 104(9), 2858-71.

Bergin, J., And W. B. MacLeod (1993): "Continuous Time Repeated Games," International Economic Review, 34(1), 21-37.

Bismut, J.-M. (1973): "Conjugate Convex Functions in Optimal Stochastic Control," Journal of Mathematical Analysis and Applications, 44(2), 384-404.

Bohren, A. (2014): "Stochastic Games in Continuous Time: Persistent Actions in LongRun Relationships," mimeo.

Budd, C., C. Harris, And J. Vickers (1993): "A Model of the Evolution of Duopoly: Does the Asymmetry between Firms Tend to Increase or Decrease?," Review of Economic Studies, 60(3), 543-573.

Calcagno, R., Y. Kamada, S. Lovo, and T. Sugaya (2014): "Asynchronicity and Coordination in Common and Opposing Interest Games," Theoretical Economics, 9, 409-434.

CAO, D. (2014): "Racing under Uncertainty: A Boundary Value Problem Approach," Journal of Economic Theory, 151, 508-527.

Carlsson, H., and E. van Damme (1993): "Global Games and Equilibrium Selection," Econometrica, 61(5), 989-1018.

Caruana, G., And L. Einav (2008a): "Production targets," RAND Journal of Economics, 39(4), 990-1017.

- (2008b): "A Theory of Endogenous Commitment," Review of Economic Studies, $75(1), 99-116$.

Chen, Z., And L. G. Epstein (2002): “Ambiguity, Risk, and Asset Returns in Continuous Time," Econometrica, 70(4), 1403-1443.

Chen, Z., R. Kulperger, And G. Wei (2005): "A Comonotonic Theorem for BSDEs," Stochastic Processes and their Applications, 115(1), 41-54.

Cheridito, P., AND K. NAm (Forthcoming): "Multidimensional Quadratic and Subquadratic BSDEs with Special Structure," Stochastics.

Compte, O., And P. Jehiel (2004): "Gradualism in Bargaining and Contribution Games," Review of Economic Studies, 71(4), 975-1000. 
Cvitanic, J., And G. Georgiadis (2015): "Achieving Efficiency in Dynamic Contribution Games," mimeo.

Cvitanic, J., X. Wan, And J. Zhang (2009): "Optimal Compensation with Hidden Action and Lump-Sum Payment in a Continuous-Time Model," Applied Mathematics and Optimization, 59(1), 99-146.

Cvitanic, J., And J. Zhang (2012): Contract Theory in Continuous-Time Models. Springer Finance.

Delarue, F. (2002): "On the Existence and Uniqueness of Solutions to FBSDEs in a Non-degenerate Case," Stochastic Processes and their Applications, 99(2), 209-286.

DeMarzo, P. M., And Y. Sannikov (2006): "Optimal Security Design and Dynamic Capital Structure in a Continuous-Time Agency Model," Journal of Finance, 61(6), 2681-2724.

Dockner, E. J., S. Jorgensen, N. V. Long, And G. Sorger (2001): Differential Games in Economics and Management Science. Cambridge University Press.

Dos Reis, G., And R. J. Dos Reis (2013): “A Note on Comonotonicity and Positivity of the Control Components of Decoupled Quadratic FBSDE," Stochastics and Dynamics, 13(4), 1350005.

Duffie, D., And L. G. Epstein (1992): “Stochastic Differential Utility," Econometrica, $60(2), 353-394$.

Faingold, E., And Y. SAnnikov (2011): "Reputation in Continuous-Time Games," Econometrica, 79(3), 773-876.

Fershtman, C., And M. I. Kamien (1987): "Dynamic Duopolistic Competition with Sticky Prices," Econometrica, 55(5), 1151-1164.

Fershtman, C., And S. Nitzan (1991): "Dynamic Voluntary Provision of Public Goods," European Economic Review, 35(5), 1057-1067.

Fudenberg, D., And D. Levine (2007): "Continuous Time Limits of Repeated Games with Imperfect Public Monitoring," Review of Economic Dynamics, 10(2), 173-192.

- (2009): "Repeated Games with Frequent Signals," Quarterly Journal of Economics, 124, 233-265.

FudenberG, D., And J. Tirole (1983): "Capital as a Commitment: Strategic Invest- 
ment to Deter Mobility," Journal of Economic Theory, 31(2), 227-250.

Georgiadis, G. (2015): "Projects and Team Dynamics," Review of Economic Studies, 82(1), 187-218.

Gobet, E., J.-P. Lemor, And X. Warin (2005): "A Regression-based Monte Carlo Method to Solve Backward Stochastic Differential Equations," Annals of Applied Probability, 15(3), 2172-2202.

GuÉron, Y. (2015): "Failure of Gradualism under Imperfect Monitoring," Journal of Economic Theory, 157, 128-145.

Hamadene, S., And R. Mu (2015): "Existence of Nash Equilibrium Points for Markovian Non-zero-sum Stochastic Differential Games with Unbounded Coefficients," Stochastics, 87(1), 85-111.

Harris, C., AND J. Vickers (1987): "Racing with Uncertainty," Review of Economic Studies, 54(1), 1-21.

Iijima, R., And A. Kasahara (2015): "Unique Equilibrium Selection and Potential: A Continuous-Time Approach," mimeo.

Kamada, Y., And M. Kandori (2014): "Revision Games," mimeo.

Karatzas, I., And S. E. Shreve (1991): Brownian Motion and Stochastic Calculus (Graduate Texts in Mathematics). Springer.

Kobylanski, M. (2000): "Backward Stochastic Differential Equations and Partial Differential Equations with Quadratic Growth," Annals of Probability, 28(2), 558-602.

Konrad, K. A. (2009): Strategy and Dynamics in Contests. Oxford University Press.

Ladyzenskaja, O. A., V. A. Solonnikov, and N. N. Ural'Ceva (1995): Linear and Quasi-linear Equations of Parabolic Type. American Mathematical Society.

Lepeltier, J.-P., Z. Wu, AND Z. Yu (2009): "Nash Equilibrium Point for One Kind of Stochastic Nonzero-sum Game Problem and BSDEs," Comptes Rendus Mathematique, 347, 959-964.

Lipman, B. L., AND R. WANG (2000): "Switching Costs in Frequently Repeated Games," Journal of Economic Theory, 93(2), 148-90.

LockWood, B. (1996): "Uniqueness of Markov-perfect equilibrium in infinite-time affinequadratic differential games," Journal of Economic Dynamics and Control, 20(5), 751- 
765.

Lockwood, B., And J. Thomas (2002): "Gradualism and Irreversibility," Review of Economic Studies, 69, 339-356.

Lovo, S., And T. Tomala (2015): "Markov Perfect Equilibria in Stochastic Revision Games," mimeo.

Marx, L., And S. Matthews (2000): "Dynamic Voluntary Contribution to a Public Project," Review of Economic Studies, 67, 327-358.

Matthews, S. A. (2013): "Achievable Outcomes of Dynamic Contribution Games," Theoretical Economics, 8(2), 365-403.

Papavassilopoulos, G. P., And J. B. Cruz (1979): "On the Uniqueness of Nash Strategies for a Class of Analytic Differential Games," Journal of Optimization Theory and Applications, 27(2), 309-314.

Pardoux, E., And S. Peng (1990): "Adapted Solution of a Backward Stochastic Differential Equation," Systems and Control Letters, 14(1), 55-61.

Pham, H. (2010): Continuous-time Stochastic Control and Optimization with Financial Applications. Springer.

Pitchford, R., And C. Snyder (2004): "A Solution to the Hold-up Problem involving Gradual Investment," Journal of Economic Theory, 114, 88-103.

RADner, R. (1992): "Hierarchy: The Economics of Managing," Journal of Economic Literature, 30(3), 1382-1415.

Sannikov, Y. (2007): "Games with Imperfectly Observable Actions in Continuous Time," Econometrica, 75(5), 1285 - 1329.

Sannikov, Y., AND A. SkrzyPaCz (2007): "Impossibility of Collusion under Imperfect Monitoring with Flexible Production," American Economic Review, 97(5), 1794-1823. (2010): "The Role of Information in Repeated Games with Frequent Actions," Econometrica, 78(3), 847-882.

Seel, C., And P. Strack (Forthcoming): "Continuous Time Contests with Private Information," Mathematics of Operations Research.

Simon, L. K., And M. B. Stinchcombe (1989): "Extensive Form Games in Continuous Time: Pure Strategies," Econometrica, 57(5), 1171-1214. 
Spence, A. M. (1979): "Investment Strategy and Growth in a New Market," Bell Journal of Economics, 10(1), 1-19.

Tsutsui, S., And K. Mino (1990): "Nonlinear Strategies in Dynamic Duopolistic Competition with Sticky Prices," Journal of Economic Theory, 52, 136-161.

Wernerfelt, B. (1987): "Uniqueness of Nash Equilibrium for Linear-Convex Stochastic Differential Game," Journal of Optimization Theory and Applications, 53(1), 133-138.

Williams, N. (2011): "Persistent Private Information," Econometrica, 79(4), 1233-1275.

Yildirim, H. (2005): "Contests with Multiple Rounds," Games and Economic Behavior, $51,213-227$.

Zandt, T. V. (1999): "Decentralized Information Processing in the Theory of Organizations," in Contemporary Economic Issues: Economic Behaviour and Design, ed. by M. Sertel, vol. 4, chap. 7, pp. 125-160. Macmillan. 